\title{
The Meso-NH Atmospheric Simulation System. Part I: adiabatic formulation and control simulations
}

\author{
J. P. Lafore ${ }^{1}$, J. Stein ${ }^{1}$, N. Asencio ${ }^{1}$, P. Bougeault ${ }^{1}$, V. Ducrocq ${ }^{1}$, J. Duron ${ }^{2}$, C. Fischer $^{1}$, P. Héreil ${ }^{1}$, P. Mascart ${ }^{2}$, \\ V. Masson $^{1}$ J. P. Pinty ${ }^{2}$, J. L. Redelsperger ${ }^{2}$, E. Richard ${ }^{2}$, J. Vilà-Guerau de Arellano ${ }^{2}$ \\ ${ }^{1}$ Centre National de Recherches Météorologiques F-31057 Toulouse, France \\ ${ }^{2}$ Laboratoire d'Aérologie, F-31400 Toulouse, France
}

Received: 20 July 1996 / Revised: 14 May 1997 / Accepted: 2 June 1997

\begin{abstract}
The Meso-NH Atmospheric Simulation System is a joint effort of the Centre National de Recherches Météorologiques and Laboratoire d'Aérologie. It comprises several elements; a numerical model able to simulate the atmospheric motions, ranging from the large meso-alpha scale down to the micro-scale, with a comprehensive physical package, a flexible file manager, an ensemble of facilities to prepare initial states, either idealized or interpolated from meteorological analyses or forecasts, a flexible post-processing and graphical facility to visualize the results, and an ensemble of interactive procedures to control these functions. Some of the distinctive features of this ensemble are the following: the model is currently based on the Lipps and Hemler form of the anelastic system, but may evolve towards a more accurate form of the equations system. In the future, it will allow for simultaneous simulation of several scales of motion, by the so-called "interactive grid-nesting technique". It allows for the in-line computation and accumulation of various terms of the budget of several quantities. It allows for the transport and diffusion of passive scalars, to be coupled with a chemical module. It uses the relatively new Fortran 90 compiler. It is tailored to be easily implemented on any UNIX machine. Meso-NH is designed as a research tool for small and meso-scale atmospheric processes. It is freely accessible to the research community, and we have tried to make it as "user-friendly" as possible, and as general as possible, although these two goals sometimes appear contradictory. The present paper presents a general description of the adiabatic formulation and some of the basic validation simulations. A list of the currently available physical parametrizations and initialization methods is also given. A more precise description of these aspects will be provided in a further paper.
\end{abstract}

Correspondence to: P. Bougeault (philippe.bougeault@meteo.fr)

\section{Introduction}

Numerical models for research on atmospheric processes are becoming increasingly complex tools as the considered physical processes grow in number and complexity and as the range of scales of interest expands. Some years ago, individual scientists could develop their own model and obtain original results in any specific domain at an interesting quality-price ratio. This is no longer true. To cite only one example, cloud resolving models have achieved in recent years a high degree sophistication and realism, and may now challenge climate models in complexity. On the other hand, the operational forecasting models of the National Weather Services often lack the flexibility required for research tools. There is therefore a definite need for community research models.

In France, three models have been developed and used independently for the last 15 years. The research version of the PERIDOT limited-area model of the CNRM (Bougeault et al., 1991) is a hydrostatic, primitive equation model in sigma vertical coordinate, with a complete but simple physical package. The SALSA model (Nickerson et al., 1986) developed at the LA, shares many characteristics with the previous one, but has different physics. Finally CNRM has developed a cloudresolving model, also allowing for large eddy simulations (Redelsperger and Lafore, 1988). This model does not account for orography or surface processes. A consensus was reached in the French community of meso- and micro-scale modellers that rather than continuing to develop three different tools, it would be more efficient to start a new project, and develop a single, all-purpose model. This model should have the capacity to represent all dynamical and physical processes of interest to our community, at a reasonable quality-cost ratio. It should be user-friendly, in order to allow rapid acquaintance for new users and students. It should be easily adaptable to a large number of sites. We conducted a detailed review of 
the needs of the community and of the available facilities. As a direct outcome, the Meso-NH model was developed by a dedicated team of scientists during the period 1994 1997. We present here the main original aspects of the model formulation, and some of the control simulations, which are currently used to access its quality. It is by no means possible to present an exhaustive account of the model in such a paper, and the reader is referred to the Model documentation for more details (Bougeault et al., 1996).

The Meso-NH model is comparable to some extent to several contemporary and widely used models, and we have tried to build on these experiences and develop a fine working tool. They are mainly (1) the anelastic model of Clark (1977), with its interactive grid-nesting capability; (2) the MM5 model (Dudhia, 1993); (3) the ARPS model (Xue et al., 1995); and (4) the Canadian MC2 model (Benoit et al., 1997). Most of these models have in common with Meso-NH some objectives of flexibility, portability and maintainability.

Among the main differences between similar models, the choice of the equation system and the timediscretization technique is generally considered an important topic. In the frame of the Meso-NH project, we conducted a number of preliminary studies to evaluate the various existing solutions, ranging from the time-splitting or semi-implicit methods in the case of the full compressible system, to the several forms of the anelastic approximation. We concluded in favour of the anelastic approach, at least for the first part of the project. A beneficial effect of the anelastic approximation is that the system has no acoustic modes, and the time discretization maybe entirely explicit without introducing an overly restrictive constraint on the time-step. Other advantages are the very simple formulation of the interaction between the dynamics and the physics, especially for the condensation of water, and the possibility to carry a Boussinesq form of the equations. We also thought that the development of the grid-nesting technique would be facilitated by the anelastic approximation. Finally, our good experience with the anelastic system was considered an asset.

This choice led us to several original developments: an additional equations has been introduced to allow for the absolute pressure computation, which up to now has not been possible for anelastic systems. An efficient method was developed to solve the elliptic pressure problem.

In the future, we will consider the development of a semi-implicit, compressible version of the model, which presents a similar pressure problem to filter out acoustic waves. A semi-implicit algorithm for internal gravity waves is also under development.

\section{Basic equations in Cartesian frame}

\subsection{Prognostic variables and large-scale values}

Left us first define a few notations. The basic atmospheric variables used are the temperature $T$, the pressure $P$, the total density of the moist air $\rho$ and the density of the dry fraction of the air $\rho_{d} \cdot \vec{U}$ is the air velocity. A more complete list of symbols may be found in the Appendix. Various substances are measured by their mixing ratio $r_{*}$, which is the mass of the substance within a given volume, divided by the mass of dry air within the same volume. In the current formulation however, we assimilate the dry-air mass and the reference state dry-air mass (see below), thus the volumic mass of any substance may be recovered as $\rho_{\text {dref }} r_{*}$.

The treatment of the different phases of water is flexible. Up to six forms of water may be considered if the user wished to do so, the mixing ratios being $r_{v}$ for vapour, $r_{c}$ for the cloud liquid water, $r_{r}$ for liquid rain, $r_{i}$ for cloud ice, $r_{s}$ for snow and $r_{g}$ for graupels. If one user is not interested in such computations, he may decide to use only a subset of these variables. The computations concerning the remaining water variables will not be performed and the computer memory will not be allocated, resulting in lower cost. The resulting equations may be obtained by setting the corresponding variables to zero in the following of this chapter. Whenever necessary, we use the mixing ratio of total water substance $r_{w}=r_{v}+r_{c}+r_{r}+r_{i}+r_{s}+r_{g}$.

An important quantity is the Exner function

$\Pi=\left(P / P_{00}\right)^{R_{d} / C_{p d}}$,

from which the "dry" potential temperature

$\theta=\frac{T}{\Pi}$

is obtained. The virtual temperature is defined as

$T_{v}=T \cdot\left(1+r_{v} R_{v} / R_{d}\right) /\left(1+r_{w}\right)$,

and the virtual potential temperature as

$\theta_{v}=\frac{T_{v}}{\Pi}=\theta \cdot\left(1+r_{v} R_{v} / R_{d}\right) /\left(1+r_{w}\right)$.

The prognostic variables of the model are the three Cartesian components of the velocity $u, v, w$, the dry potential temperature $\theta$, the various water mixing ratios $r_{*}$, and, if so specified, an arbitrary number of scalars $s_{*}$, available for chemistry computations or other specific applications.

One original aspect of the model formulation is to allow a continuous interaction with "large-scale" determinations of these quantities. These "large-scale fields" are useful to initialize the model prognostic variables, to supply a realistic time-evolving boundary forcing, or to define the background diffusion operator, as well as the lateral and vertical relaxation terms. To achieve this, we carry in the model three-dimensional arrays called, respectively, $u_{L S}, v_{L S}, w_{L S}, \theta_{L S}$ and $r_{* L S}$. They are provided by analysis or forecast from larger-scale simulation systems (e.g. the operational products of Météo-France or the ECMWF), and may evolve in time if so chosen by the user, For more idealized studies, these fields may be specified from analytical expressions, or even set to zero. 


\subsection{Reference state}

The present formulation of the model is based on the Lipps and Hemler (1982) form of the anelastic approximation. This relies on the assumption that the atmosphere will not depart very much from a "reference state" having a potential temperature varying slowly in the vertical. Under this same constraint, we define the Meso-NH reference state as an atmosphere at rest, in hydrostatic equilibrium, with horizontally uniform profiles of temperature $T_{r e f}(z)$ and water vapour $r_{v \text { ref }}(z)$. No condensed water is considered in the reference state. For our application, the reference profiles are often chosen as the initial horizontal averages of the "largescale" fields over the expected domain of simulation. Any profile, however may be used, but the inaccuracy of the computation increases if the reference state is far from the actual mean state. In the following, the dependence on $z$ only will be assumed for all quantities subscripted with ()$_{\text {ref }}$.

At the beginning of each experiment, the properties of the reference state are computed in the following way: the hydrostatic relation and the equation of state are used to derive the profiles of the virtual temperature $T_{\text {vref }}$ and the Exner function $\Pi_{r e f}$ of the reference state, as

$T_{v r e f}=T_{\text {ref }}\left(1+r_{v r e f} R_{v} / R_{d}\right) /\left(1+r_{v r e f}\right)$,

$\frac{\mathrm{d}\left(\log \Pi_{r e f}\right)}{\mathrm{d} z}=-\frac{g}{C_{p d} T_{v r e f}}$

with the upper boundary condition $\Pi_{r e f}=\Pi_{r e f}^{\text {top }}$ at the model top $z=H$. The virtual potential temperature $\theta_{v \text { ref }}$ is then deduced from $\Pi_{r e f}$ and $T_{v e f}$ as

$\theta_{\text {vref }}=\frac{T_{v r e f}}{\Pi_{r e f}}$.

Finally, $\rho_{\text {ref }}$ is deduced from the equation of state

$\rho_{r e f}=\frac{\Pi_{r e f}^{C_{p d} / R_{d}} P_{00}}{R_{d} T_{v r e f}}$,

and the density of the dry air fraction $\rho_{d r e f}$ is retrieved as

$\rho_{\text {dref }}=\frac{\rho_{\text {ref }}}{\left(1+r_{\text {vref }}\right)}$.

Note that both the dry air and the water vapour must be considered to build the profile of the Exner function of the reference state, since each gas is subject to the partial pressure of the other.

A Boussinesq version of the model is obtained in a very simple way, thanks to the use of the reference state. This is achieved by enforcing uniform values of $\rho_{d r e f}$, $\theta_{\text {vref }}$ and $\Pi_{\text {ref }}$ in the vertical instead of using the preceding equations. This provides exactly the same equations for the Boussinesq approximation as those derived by Sinocca and Shepperd (1992).

\subsection{Linearized equation of state}

The equation of state $p=\rho R_{d} T_{v}$ is used under its linearized form

$\rho^{\prime}=\rho_{r e f}\left(\frac{C_{v d}}{R_{d}} \frac{\Pi^{\prime}}{\Pi_{r e f}}-\frac{\theta_{v}^{\prime}}{\theta_{v r e f}}\right)$,

where $\rho^{\prime}=\rho-\rho_{\text {ref }}, \Pi^{\prime}=\Pi-\Pi_{\text {ref }}$ and $\theta_{v}^{\prime}=\theta_{v}-\theta_{v \text { ref }}$. For convenience, the model uses $\theta_{v}$ rather than $\theta_{v}^{\prime}$ (therefore, in the following equations, we will use $\theta_{v}-\theta_{v \text { ref }}$ to denote the resolved fluctuation of potential temperature).

\subsection{Anelastic constraint}

The continuity equation is used under the approximate form

$$
\vec{\nabla} \cdot\left(\rho_{\text {dref }} \vec{U}\right)=0 \text {. }
$$

It represents a strong kinematic constraint on the wind field, hereafter called the anelastic constraint. This constraint is enforced by solving the elliptic equation for the pressure function $\Phi$, that results from the combination of Eq. (11) with the momentum conservation equation (see section 2.6).

\subsection{Conservation of momentum}

The equation is written in a framework linked to the earth with rotation velocity $\vec{\Omega}$. In Meso-NH, the total density of the air may vary as a result of changes in the water content. Therefore, a consistent flux form of the equations can only be obtained by combining the advective forms and the equation of continuity for dry air. Furthermore, we want to allow for an easy transition to the compressible system, should it be adopted in the future; we therefore multiply the momentum equation by $\rho_{d r e f}$, leading to

$$
\begin{aligned}
& \frac{\partial}{\partial t}\left(\rho_{d r e f} \vec{U}\right)+\vec{\nabla} \cdot\left(\rho_{d r e f} \vec{U} \otimes \vec{U}\right)+\rho_{d r e f} \vec{\nabla} \Phi \\
& +\rho_{d r e f} \vec{g} \frac{\theta_{v}-\theta_{\text {vref }}}{\theta_{\text {vref }}}+2 \rho_{d r e f} \overrightarrow{\boldsymbol{\Omega}} \wedge \vec{U}=\rho_{d r e f} \overrightarrow{\mathscr{F}}
\end{aligned}
$$

This may be interpreted as the conservation of the dryair fraction, although it is strictly equivalent to the usual momentum equation. The different terms represent, respectively, the time evolution, the advection, the pressure gradient, the buoyancy force, the Coriolis force and the turbulence effects. Note that the flux form of the equation, which is kept consistently throughout the model, ensures an integral conservation of momentum by the advection term to a very good accuracy.

The pressure function is defined as

$\Phi=C_{p d} \theta_{v r e f} \Pi^{\prime}$

and has the same physical dimension as a geopotential. As a distinctive characteristic of the Lipps and Hemler 
formulation, it includes the reference virtual potential temperature. This allows for a better conservation of mechanical energy in the high atmosphere (see e.g. Scinocca and Shepherd, 1992; Nance and Durran, 1994).

\subsection{The pressure problem}

The determination of $\Phi$ is often called the pressure problem. In order to shorten the expression of this problem, we introduce the dynamical sources of momentum

$$
\begin{aligned}
& \vec{S}=-\vec{\nabla} \cdot\left(\rho_{\text {dref }} \vec{U} \otimes \vec{U}\right)-\rho_{d r e f} \vec{g} \frac{\theta_{v}-\theta_{\text {vref }}}{\theta_{\text {vref }}} \overrightarrow{\mathscr{F}}, \\
& -2 \rho_{\text {dref }} \overrightarrow{\boldsymbol{\Omega}} \wedge \vec{U}+\rho_{\text {dref }}
\end{aligned}
$$

whereby the momentum conservation equation may be rewritten

$\frac{\partial}{\partial t}\left(\rho_{\text {dref }} \vec{U}\right)=\vec{S}-\rho_{d r e f} \vec{\nabla} \Phi$.

By applying the anelastic constraint Eq. (11) to this equation, it becomes readily

$$
\vec{\nabla} \cdot\left(\rho_{\text {dref }} \vec{\nabla} \Phi\right)=\vec{\nabla} \cdot \vec{S}
$$

Thus, the pressure function $\Phi$ is the solution of an elliptic problem. The algorithm to solve for $\Phi$ is an important component of many non-hydrostatic models and will be discussed later.

\subsection{Thermodynamic equation}

For the time being, the prognostic energy variable is the dry potential temperature $\theta$. In order to account for the effects of moisture, its equation takes the following approximate form

$$
\begin{aligned}
& \frac{\partial}{\partial t}\left(\rho_{\text {dref }} \theta\right)+\vec{\nabla} \cdot\left(\rho_{d r e f} \theta \vec{U}\right)= \\
& -g \rho_{\text {dref }} w \frac{\theta}{C_{p d} T_{v r e f}}\left[\frac{R_{d}+r_{v} R_{v}}{R_{d}} \frac{C_{p d}}{C_{p h}}-1\right] \\
& +\frac{\rho_{d r e f}}{\prod_{\text {ref }} C_{p h}}\left[L_{m} \frac{D\left(r_{i}+r_{s}+r_{g}\right)}{D t}-L_{v} \frac{D r_{v}}{D t}+\mathscr{H}\right] .
\end{aligned}
$$

The terms on the right-hand side represent, respectively, the moist correction in absence of any phase change, the effects of phase changes and the other diabatic effects (radiation and diffusion). This equation derives from the conservation of entropy. The only approximation made is to replace the Exner function $\Pi$ by the references state Exner function $\Pi_{\text {ref }}$ on the right-hand side, and to substitute $\Pi_{\text {ref }}$ using the hydrostatic equation. This allows for an effective decoupling of the pressure problem from the thermodynamic equation leading to a great simplification of the model while retaining an excellent accuracy.

\subsection{Conservation of moisture and passive scalars}

For any of the water substances $r_{*}$, the conservation equation is written

$\frac{\partial}{\partial t}\left(\rho_{d r e f} r_{*}\right)+\vec{\nabla} \cdot\left(\rho_{d r e f} r_{*} \vec{U}\right)=\rho_{\text {dref }} \mathscr{Q}_{*}$,

where $\mathscr{Q}_{*}$ stands for the effects of phase changes, sedimentation and diffusion. Note that the flux form, and the use of $\rho_{\text {dref }}$ (not $\rho_{\text {ref }}$ ), ensure the existence of integral conservation properties.

Similarly, the model can carry an arbitrary number of passive scalars, following the equation:

$\frac{\partial}{\partial t}\left(\rho_{d r e f} s_{*}\right)+\vec{\nabla} \cdot\left(p_{d r e f} s_{*} \vec{U}\right)=\rho_{d r e f} \mathscr{S}_{*}$,

where $\mathscr{S}_{*}$ stands for the effects of diffusion and possible chemical processes.

\subsection{Boundary conditions}

2.9.1 Top boundary. The model is assumed to be limited by a rigid horizontal lid, exerting a free slip condition on the atmosphere. The height $H$ of this lid may be chosen by the user. In order to obtain realistic results, it is recommended that $H$ is placed somewhere in the stratosphere. At $z=H$, the conditions imposed on the model are:

$\vec{U} \cdot \vec{n}=0$

$\rho_{\text {dref }} \frac{\partial \Phi}{\partial n}=\vec{S} . \vec{n}$,

where $\vec{n}$ is a vertical unit vector.

In order to prevent the reflexion of gravity waves on this lid, an absorbing layer may be activated, where the model prognostic variables are relaxed towards the large-scale (LS) values.

2.9.2 Bottom boundary. In the adiabatic formulation of the model, the lower boundary is defined as an insulated rigid lid allowing for free slip tangential velocity. This is formulated in the same way as Eqs. (20)-(21), but $\vec{n}$, a unit vector normal to the earth's surface, may not be locally vertical. In the physical package of the model, the turbulent fluxes of heat, moisture and momentum normal to the surface are computed through bulk drag coefficients depending on wind and stability. This results in the near-surface velocity getting close to zero, as expected whenever the user wants to simulate realistic surface friction.

2.9.3 Lateral boundaries Several formulations for lateral boundary condition (l.b.c. hereafter) are available. First we consider the cyclic boundary condition; this widely used boundary condition is most simple to prescribe. For instance, giving the periodicity length $L_{x}$ (corre- 
sponding to the model domain width) for the $x$ direction, the condition imposed on all model variables $\alpha$ are

$\alpha(x, y, z)=\alpha\left(x+L_{x}, y, z\right)$.

It should be noted that this option implies some specific treatment in several parts of the model. First, the computation of the advection terms on the boundary is modified. Second, the pressure solver uses complex Fourier transforms, whereas for other types of 1.b.c. the solver uses cosine Fourier transforms.

Next the rigid-wall boundary condition; for a free-slip rigid wall, a mirror-type boundary condition is assumed. This reads

$\vec{U} \cdot \vec{n}=0$,

where $\vec{n}$ is a horizontal unit vector normal to the lateral boundary. It follows that the normal velocity components are zero at the lateral boundaries, whereas all other variables are symmetric relative to the boundary,

$\frac{\partial \alpha}{\partial n}=0$.

Finally the open and externally forced boundary conditions; many usual applications of the model require simultaneously to let the short-scale waves out of the domain with minimal reflexion at the boundaries, and the large-scale evolution of the variables get into the model by boundary forcing. This is achieved through the use of a mixed radiation-relaxation method which will be now described. This requires knowledge of the large-scale values of all variables (LS, see above). Note that in many cases, these LS fields are simply identical to the initial values.

There exist several variants of radiative, or open boundary conditions in the literature. For instance, some authors apply the wave-radiation boundary condition to all prognostic variables, whereas others apply it only to the normal velocity component. We found that the second approach works better for complex cases with ambient wind shear or with intense convection. For the present version for Meso-NH, we have adopted the simplest formulation giving satisfactory results from our experience. The prognostic variables are separated in two groups: on the one hand, the scalars and the tangential velocity components; on the other, the normal wind.

For the scalars and the tangential components of velocity, the formulation depends on the sign of the normal velocity: for outflow boundaries, the derivatives normal to the boundary are extrapolated from the interior. For inflow boundaries, they are linearly interpolated between the interior and the LS values.

On the other hand, the normal velocity component $u_{n}$ is computed by a mix of the Davies (1976) and Carpenter (1982) methods, already used by Redelsperger and Lafore (1988):

$\frac{\partial u_{n}}{\partial t}=\left(\frac{\partial u_{n}}{\partial t}\right)_{L S}-C^{*}\left(\frac{\partial u_{n}}{\partial x}-\left(\frac{\partial u_{n}}{\partial x}\right)_{L S}\right)-K\left(u_{n}-u_{n L S}\right)$, where the subscript $L S$ stands for large-scale value of the field and $C^{*}$ denotes the phase speed of the perturbation field $u_{n}-\left(u_{n}\right)_{L S}$. This formulation is used for both inflow or outflow conditions. It allows waves coming from the interior of the model domain to pass out freely through the boundary with minimal reflection. It also allows the large-scale flow to force the evolution of the inner domain at large time-scales. The last term corresponds to a slow relaxation towards the large-scale value, avoiding a temporal drift of the normal velocity.

Sophisticated methods may be used to evaluated the phase speed $C^{*}$, such as proposed by Orlanski (1976). As a first step however, we use the simple method proposed by Klemp and Wilhelmson (1978), reading

$C^{*}=u_{n}+C$

where $C$ is a constant phase speed chosen by the user (in the range of 20 to $50 \mathrm{~m} \mathrm{~s}^{-1}$ ).

The three types of 1.b.c. described can be specified independently for each lateral boundary, with a single limitation: if the cyclic boundary condition is chosen for one lateral boundary, the opposing-side b.c. must also be cyclic. Note in particular that it is possible to mix a wall condition on one side and an open condition of the other side in the same direction. The combination of different types of 1.b.c. offers a wide range of geometrical configurations, such as a close tank or a channel.

\subsection{Conservation of total mass and retrieval of the absolute pressure}

While developing the model, we realized that most existing anelastic models suffer from the inability to determine precisely the absolute value of the pressure. This is usually considered a minor problem, since only the pressure gradient is used in the momentum equation. However, it may be useful to retrieve the best possible estimate of the absolute pressure for thermodynamic computations, or for the sake of comparison with observations of this quantity.

The problem is linked to the fact that the continuity equation (11) is an approximate form, which no longer represents a mass budget. We therefore developed an original technique to control the mass budget of the model. The total mass inside the model domain is the sum of the mass of dry air and the total mass of water in all forms

$\mathscr{M}=\mathscr{M}_{d}+\mathscr{M}_{w}$.

The anelastic constraint given by Eq. (11) does not supply the variation of the total mass of dry air $\mathscr{M}_{d}(t)$ inside the model domain, since it uses the reference density profile instead of the actual density. It is therefore necessary, to use an additional equation; this depends on the general conditions of the experiment. If periodic or wall boundary conditions are assumed, the total mass of dry air may not vary during a simulation. It is therefore specified once and for all at the beginning $\left(\mathscr{M}_{d}(t)=\mathscr{M}_{d}\left(t_{0}\right)\right)$. If the model is driven by larger-scale meteorological information (forecasts or analyses), the 
time variation of the total mass of dry air is assumed to be entirely governed by the larger-scale fields. This is consistent with procedures currently used in other limited area models. In that case, the value $\mathscr{M}_{d}(t)$ of the total mass of dry air within the simulation volume is regularly updated by linear interpolation in time between the values deduced from the larger-scale fields.

On the other hand, the total mass of water (in all forms) $\mathscr{M}_{w}(t)$ can be computed at each time during the simulation, by a simple volume integration

$$
\mathscr{M}_{w}(t)=\int_{V} \rho_{d r e f} r_{w} \mathrm{~d} V,
$$

where $V$ is the total volume of the model domain. Note that this results in the total mass varying with precipitation and ground evaporation of water, as it should, behaviour that was generally ignored in most models in the past. Also, when the model is forced by larger-scale field, the evolution of the total mass of water in the model may be different from that in the forcing fields, since the representation of physical processes is different.

The retrieval of the absolute pressure proceeds as follows. Let us assume that $\Phi_{f g}$ (first-guess) is a particular solution of the preceding elliptic problem. We want to determine the additional constant $\Phi_{0}$ that will ensure the correct absolute value of the pressure. This is achieved by using the total mass as just defined, which may be developed following

$$
\begin{aligned}
& \mathscr{M}(t)=\int_{V} \rho \mathrm{d} V=\int_{V} \rho_{r e f} \mathrm{~d} V+\int_{V} \rho^{\prime} \mathrm{d} V \\
& =\mathscr{M}_{r e f}+\int_{V} \rho^{\prime} \mathrm{d} V
\end{aligned}
$$

where $\mathscr{M}_{\text {ref }}$ is the mass of the references state (including both dry air and water), computed once and for all at the beginning of each experiment.

Using the linearized equation of state [Eq. (10)] and the definition of $\Phi$ [Eq. (13)], we then have

$$
\begin{aligned}
& \mathscr{M}(t)=\mathscr{M}_{r e f} \\
& +\int_{V} \rho_{r e f}\left(\frac{C_{v d}}{R_{d}} \frac{1}{C_{p d} \theta_{v r e f}} \frac{\Phi_{f g}+\Phi_{0}}{\Pi_{r e f}}-\frac{\theta_{v}-\theta_{v r e f}}{\theta_{v r e f}}\right) \mathrm{d} V,
\end{aligned}
$$

which is solved as

$$
\Phi_{0}=\frac{\mathscr{M}(t)-2 \times \mathscr{M}_{r e f}+\int_{V} \frac{\rho_{\text {ref }}}{\theta_{v r e f}}\left(\theta_{v}-\frac{C_{v d}}{R_{d}} \frac{\Phi_{f g}}{C_{p d} \Pi_{r e f}}\right) \mathrm{d} V}{\int_{V} \frac{\rho_{\text {ref }}}{\theta_{v r e f}} \frac{C_{v d}}{R_{d}} \frac{1}{C_{p d} \Pi_{r e f}} \mathrm{~d} V} .
$$

The resulting field of $\Phi_{f g}+\Phi_{0}$ is used to retrieve the absolute pressure

$$
P=P_{00}\left(\Pi_{r e f}+\frac{\Phi_{f g}+\Phi_{0}}{C_{p d} \theta_{v r e f}}\right)^{C_{p d} / R_{d}}
$$

\subsection{Some additional features}

The model may be used in two-dimensional (2D) or even one-dimensional (1D) form. These simplified formulations are obtained by omitting part of the preceding equations (e.g. the advection terms or the pressure gradient). They share with the standard $3 \mathrm{D}$ formulation nearly all routines and lines of code. Thus, we avoid redundancy in the code, we simplify the maintenance and we allow for maximum testing of the 3D code through the 2D and 1D options.

The model also includes several numerical diffusion and relaxation operators. The background diffusion operator is a fourth-order diffusion acting along the coordinate lines of the model. This operator applies to the fields of fluctuations, i.e. the departures from the large-scale values $\left(\phi-\phi_{L S}\right.$, where $\phi$ is any of the prognostic variables). The characteristic time-scale for damping of the $2 \Delta x$ waves may be chosen by the user. A lateral sponge zone and a top absorbing layer are also defined in terms of fluctuations from the LS fields.

\section{Coordinate systems and grid stretching}

In general, it is not possible to use the equations of the previous section in a simple Cartesian coordinate system, because of earth's sphericity and the underlying topography. Furthermore, it is often desirable to introduce some stretching of the coordinates to gain efficiency in representing certain atmospheric features. We will therefore use a coordinate transformation as described in this section.

\subsection{Coordinate transformation}

In meteorology, a natural coordinate system is defined by the longitude $\lambda$, the latitude $\varphi$ and the distance from the earth's centre $r$ (or the altitude above sea surface $z=r-a$, where $a$ is the earth's radius). The vector basis associated with this natural system will be called $\left(\overrightarrow{i_{0}}, \overrightarrow{j_{0}}, \vec{k}\right) ; \vec{i}_{0}$ points towards the east, $\vec{j}_{0}$ towards the north and $\vec{k}$ is vertical. In Meso-NH, we prefer to work with a conformal projection allowing for rotation with respect to this natural basis. This allows more flexibility in the direction of the coordinate lines when studying particular processes. Three types of conformal projections are supported in Meso-NH: Lambert, Polar Stereographic and Mercator. They differ mainly in their conicity parameter $K$, which varies from 0 for Mercator projection, to 1 for sterographic projections, with all values between 0 and 1 defining the family of Lambert projections. 
We use a system of curvilinear coordinates $\hat{x}, \hat{y}, \hat{z}$, defined in the following manner. On the horizontal, $\hat{x}$ and $\hat{y}$ are the distances counted from an arbitrary origin $\left(\lambda_{0}, \phi_{0}\right)$ in two arbitrary orthogonal directions on a conformal surface of projection. The traces on the sphere of these coordinate lines define two orthogonal directions in each point. We will call hereafter $\vec{i}$ and $\vec{j}$ the horizontal unit length vectors parallel to those directions. We note $\gamma$ the angle from $\vec{i}$ to $\vec{i}_{0}$ (see Fig. 1). At point $\left(\lambda_{0}, \phi_{0}\right), \gamma=-\beta$, the parameter $\beta$ being chosen by the user. At other points, the angle $\gamma$ will in general vary with $\hat{x}$ and $\hat{y}$ (because of the earth's sphericity) according to $\gamma=K\left(\lambda-\lambda_{0}\right)-\beta$. Clearly, $\vec{i}, \vec{j}$ and $\vec{k}$ form a local, Cartesian basis allowing us to represent the wind velocity vector. In the following, we will call $u, v, w$ the components of $\vec{U}$ on this basis:

$\vec{U}=u \vec{i}+v \vec{j}+w \vec{k}$.

It is the most natural decomposition of the wind in a local basis. The map scale factor is defined as the ratio of distances on the projection surface to distances on the sphere and will be called $m$.

On the vertical, we use the Gal-Chen and Sommerville (1975) coordinate

$\hat{Z}=H \frac{z-z_{s}}{H-z_{s}}$

where $H$ is the height of the model top and $z_{s}$ the height of the local topography. Since the transformation is purely vertical, $\vec{k}$ is also parallel to the $\vec{z}_{0}$ lines in the physical space (see Fig. 2).

Coordinate stretching is achieved by introducing a new system denoted $(\bar{x}, \bar{y}, \bar{z})$, and related to $\hat{x}, \hat{y}, \hat{z}$ by

$\mathrm{d} \bar{x}=\frac{\mathrm{d} \hat{x}}{\mathscr{D}_{x}(\bar{x})}$,

$\mathrm{d} \bar{y}=\frac{\mathrm{d} \hat{y}}{\mathscr{D}_{y}(\bar{y})}$,

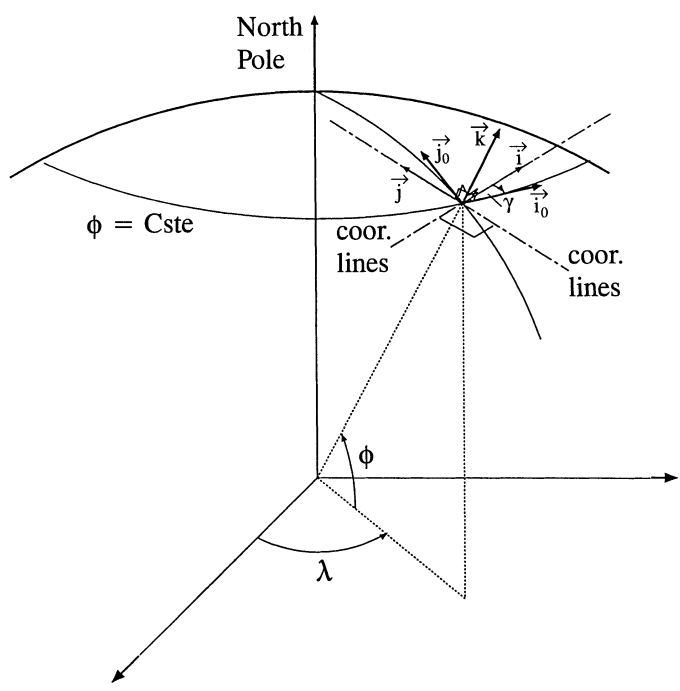

Fig. 1. Principles of projection and notation on the sphere

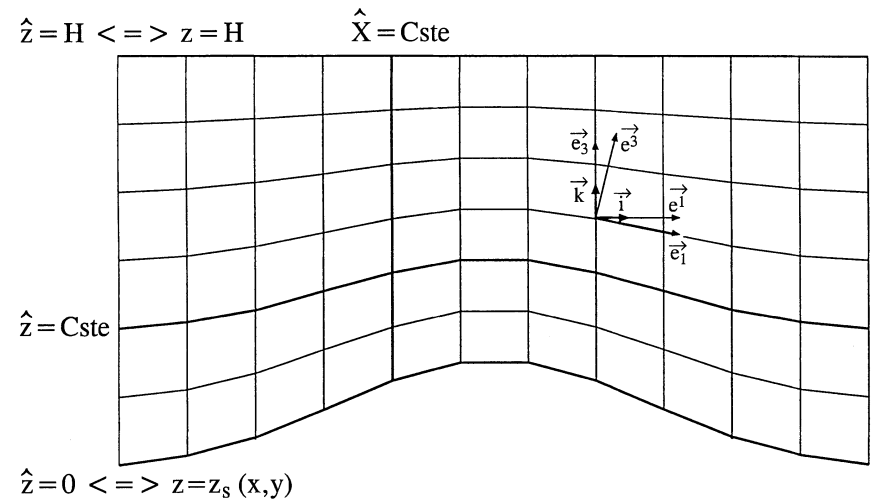

Fig. 2. The Gal-Chen and Sommerville vertical coordinate system and the Cartesian $(\vec{i}, \vec{k})$, covariant $\left(\vec{e}_{i}\right)$ and contravariant $\left(\vec{e}^{i}\right)$ bases

$\mathrm{d} \bar{z}=\frac{\mathrm{d} \hat{z}}{\mathscr{D}_{z}(\bar{z})}$.

The stretching functions $\mathscr{D}_{x}(\bar{x}), \mathscr{D}_{y}(\bar{y}), \mathscr{D}_{z}(\bar{z})$ are defined independently in the three directions.

In the physical space, the $\bar{x}, \bar{y}$ and $\bar{z}$ coordinate lines are not orthogonal, because of the underlying topography $z_{s}$. Therefore, the coordinate system is not orthogonal and it is necessary to introduce, besides the Cartesian basis, the covariant and contravariant bases to develop the operators of the previous section (see Fig. 2). Following Viviand (1974), we use both of these bases and achieve a simple formulation by retaining the Cartesian components of the velocity $u, v, w$ as prognostic variables. In order to save space, we will only give here the results of these computations.

Let us call $x, y, z$ the local distances on the sphere in the directions $\vec{i}, \vec{j}, \vec{k}$. We use the classical notation $d_{* *}$ for the metric coefficients. Their values are given by

$\mathrm{d}_{x x}=\frac{\partial x}{\partial \bar{x}}=\frac{r}{a m} \mathscr{D}_{x}(\bar{x})$,

$\mathrm{d}_{y y}=\frac{\partial y}{\partial \bar{y}}=\frac{r}{a m} \mathscr{D}_{y}(\bar{y})$,

$\mathrm{d}_{z z}=\frac{\partial z}{\partial \bar{z}}=\left(1-\frac{z_{s}}{H}\right) \mathscr{D}_{z}(\bar{z})$,

$\mathrm{d}_{z x}=\frac{\partial z}{\partial \bar{x}}=\frac{\partial z_{s}}{\partial \bar{x}}\left(1-\frac{\hat{z}}{H}\right) \mathscr{D}_{x}(\bar{x})$,

$\mathrm{d}_{z y}=\frac{\partial z}{\mathrm{~d} \bar{y}}=\frac{\partial z_{s}}{\partial y}\left(1-\frac{\hat{z}}{H}\right) \mathscr{D}_{y}(\bar{y})$,

while the other $d_{* *}$ are zero.

The Jacobian of the transformation from $\bar{x}, \bar{y}, \bar{z}$ to $x$, $y, z$ is given by

$J=\mathrm{d}_{x x} \mathrm{~d}_{y y} \mathrm{~d}_{z z}=\left(\frac{r}{a m}\right)^{2}\left(1-\frac{z_{s}}{H}\right) \mathscr{D}_{x}(\bar{x}) \mathscr{D}_{y}(\bar{y}) \mathscr{D}_{z}(\bar{z})$. 
This is the ratio of the volumes in the physical and transformed spaces.

The expression of the divergence operator in the stretched coordinate system dictates the choice for the model prognostic variables as the product of the wind Cartesian components, potential temperature and mixing ratios of all scalars by the dry density of the references state, and by the Jacobian J. To simplify the notation, we put $\tilde{\rho}=\rho_{\text {dref }} J$. The prognostic variables of the model are therefore $\tilde{\rho} u, \tilde{\rho} v, \tilde{\rho} w, \tilde{\rho} \theta, \tilde{\rho} r *$ and $\tilde{\rho} s_{*}$. In practice, the values of $\bar{x}, \bar{y}, \bar{z}$ are 1 on the first grid point, 2 on the second etc., and $\mathscr{D}_{x}(\bar{x}), \mathscr{D}_{y}(\bar{y}), \mathscr{D}_{z}(\bar{z})$ are equal to the local values of the grid size on the plane of projection. Therefore, $\mathrm{d}_{x x}, \mathrm{~d}_{y y}$ and $\mathrm{d}_{z z}$ are exactly the horizontal and vertical grid sizes in the physical space $J$ is the volume of the grid cell, and $\tilde{\rho}$ is the mass of dry air of the reference state within each grid cell. For the water and passive scalars, the prognostic variable represents therefore (to the extent that $\rho_{d r e f}$ is a good approximation of $\rho_{d}$ ) the mass of substances within the grid cell, which is very convenient to guarantee the conservation of mass.

Finally, the model equations use extensively the contravariant components of the velocity vector. These are computed at the beginning of each time-step as

$$
\begin{aligned}
U^{c} & =\frac{u}{\mathrm{~d}_{x x}}, \\
V^{c} & =\frac{v}{\mathrm{~d}_{y y}}, \\
W^{c} & =\frac{w}{\mathrm{~d}_{z z}}-\frac{u \mathrm{~d}_{z x}}{\mathrm{~d}_{x x} \mathrm{~d}_{z z}}-\frac{v \mathrm{~d}_{z y}}{\mathrm{~d}_{y y} \mathrm{~d}_{z z}} .
\end{aligned}
$$

\subsection{Approximations}

Before giving the final form of the equations, it is useful to introduce two possible approximations, controlled by flags in the Meso-NH code. The depth of the atmosphere $(\simeq 30 \mathrm{~km})$ is much smaller than the earth's radius $(a \simeq 6000 \mathrm{~km})$. It is therefore customary in meteorology to assume that the atmosphere is a thin shell $(z \ll a$ and $r \simeq a$ ). However, if this assumption is retained without care, the principle of conservation of angular momentum may be violated (Phillips, 1966). It is necessary to make the additional hypothesis that the horizontal component of the earth's rotation is negligible. In the Meso-NH model, we decided not to retain the thin-shell approximation. However, for the purpose of comparison with models making this approximation, we introduce a flag $\delta_{1}$, taking the value 1 in the general case and 0 in the case of the thin-shell approximation.

For many purposes, one may want to work in the much simpler Cartesian frame on a plane tangent to the sphere, and neglect the curvature terms (e.g. study of very small scale processes, or idealized studies of mesoscale processes). This may be obtained easily by setting $f=2 \Omega \sin \varphi_{0}, f^{*}=2 \Omega \cos \varphi_{0}, K=0, m=1, r=a=\infty$. Note that contrary to the previous case, we may here retain $f^{*} \neq 0$ without violating the conservation of angular momentum. In order to obtain this very simple form, we introduce another flag $\delta_{2}$ in the general system, taking the value 1 for the general case, and 0 for the Cartesian case.

The combination of the thin-shell approximation and the Cartesian framework results in the traditional $f$ plane approximation (no horizontal component of the earth's rotation).

\subsection{Final form of equations before discretization}

The final set of equations in the stretched, terrainfollowing coordinate system, using the $\delta_{1}$ and $\delta_{2}$ flags, is now given. For clarity, let us recall that $\phi$ is the current latitude, $K$ is the conicity parameter of the projection and $\gamma$ is the current value of the angle between the $\bar{x}$ coordinate line and the east direction. The distance from the earth's centre is $r=a+\delta_{1} \delta_{2} z$.

The anelastic constraint reads

$\frac{\partial}{\partial \bar{x}}\left(\tilde{\rho} U^{c}\right)+\frac{\partial}{\partial \bar{y}}\left(\tilde{\rho} V^{c}\right)+\frac{\partial}{\partial \bar{z}}\left(\tilde{\rho} W^{c}\right)=0$.

The conservation of momentum reads

$$
\begin{aligned}
\frac{\partial}{\partial t}(\tilde{\rho} u)= & -\frac{\partial}{\partial \bar{x}}\left(\tilde{\rho} U^{c} u\right)-\frac{\partial}{\partial \bar{y}}\left(\tilde{\rho} V^{c} u\right)-\frac{\partial}{\partial \bar{z}}\left(\tilde{\rho} W^{c} u\right) \\
& +\delta_{2} \tilde{\rho} u v \frac{\cos \gamma}{r \cos \varphi}(\sin \varphi-K) \\
& +\delta_{2} \tilde{\rho} v^{2} \frac{\sin \gamma}{r \cos \varphi}(\sin \varphi-K) \\
& -\delta_{2} \delta \delta_{1} \tilde{\rho} \frac{u w}{r}-\tilde{\rho} \frac{1}{\mathrm{~d}_{x x}} \frac{\partial \Phi}{\partial \bar{x}}+\tilde{\rho} \frac{\mathrm{d}_{x x}}{\mathrm{~d}_{x x} \mathrm{~d}_{z z}} \frac{\partial \Phi}{\partial \bar{z}} \\
& -\delta_{1} \tilde{\rho} f^{*} \cos \gamma w+\tilde{\rho} f v+\tilde{\rho} \vec{F}_{v} \cdot \vec{i} \\
\frac{\partial}{\partial t}(\tilde{\rho} v)= & \frac{\partial}{\partial \bar{x}}\left(\tilde{\rho} U^{c} v\right)-\frac{\partial}{\partial \bar{y}}\left(\tilde{\rho} V^{c} v\right)-\frac{\partial}{\partial \bar{z}}\left(\tilde{\rho} W^{c} v\right) \\
& -\delta_{2} \tilde{\rho} u^{2} \frac{\cos \gamma}{r \cos \varphi}(\sin \varphi-K) \\
& -\delta_{2} \tilde{\rho} u v \frac{\sin \gamma}{r \cos \varphi}(\sin \varphi-K) \\
& -\delta_{2} \delta_{1} \tilde{\rho} \frac{v w}{r}-\tilde{\rho} \frac{1}{\mathrm{~d}_{y y}} \frac{\partial \Phi}{\partial \bar{y}}+\tilde{\rho} \frac{\mathrm{d}_{z y}}{\mathrm{~d}_{y y} \mathrm{~d}_{z z}} \frac{\partial \Phi}{\partial \bar{z}} \\
& -\delta_{1} \tilde{\rho} f^{*} \sin \gamma w-\tilde{\rho} f u+\tilde{\rho} \vec{F}_{v} \cdot \vec{j} .
\end{aligned}
$$




$$
\begin{aligned}
\frac{\partial}{\partial t}(\tilde{\rho} w) & =-\frac{\partial}{\partial \bar{x}}\left(\tilde{\rho} U^{c} w\right)-\frac{\partial}{\partial \bar{y}}\left(\tilde{\rho} V^{c} w\right)-\frac{\partial}{\partial \bar{z}}\left(\tilde{\rho} W^{c} w\right) \\
& +\delta_{2} \delta_{1} \tilde{\rho} \frac{u^{2}+v^{2}}{r}-\tilde{\rho} \frac{1}{\mathrm{~d}_{z z}} \frac{\partial \Phi}{\partial \bar{z}}+\tilde{\rho} g \frac{\theta_{v}^{\prime}}{\bar{\theta}_{v}} \\
& +\delta_{1} \tilde{\rho} f^{*}(\sin \gamma v+\cos \gamma u)+\tilde{\rho} \vec{F}_{v} \cdot \vec{k} .
\end{aligned}
$$

The thermodynamic equation reads

$$
\begin{aligned}
\frac{\partial}{\partial t}(\tilde{\rho} \theta)= & -\frac{\partial}{\partial \bar{x}}\left(\tilde{\rho} U^{c} \theta\right)-\frac{\partial}{\partial \bar{y}}\left(\tilde{\rho} V^{c} \theta\right)-\frac{\partial}{\partial \bar{z}}\left(\tilde{\rho} W^{c} \theta\right) \\
& -\tilde{\rho} g w \frac{\theta}{\prod_{r e f} \theta_{v r e f} C_{p d}}\left[\frac{R_{d}+r_{v} R_{v}}{R_{d}} \frac{C_{p d}}{C_{p h}}-1\right] \\
& +\frac{\tilde{\rho}}{\prod_{r e f} C_{p h}}\left[L_{m} \frac{D\left(r_{i}+r_{s}+r_{g}\right)}{D t}-L_{v} \frac{D r_{v}}{D t}+\mathscr{H}\right] .
\end{aligned}
$$

The equations for moisture and additional scalars take similar forms.

\subsection{Discretization}

We use a C-grid in the Arakawa and Mesinger (1976) terminology both in the horizontal and in the vertical (Fig. 3); $\hat{x}, \hat{y}, \hat{z}$ and the metric coefficients $d_{x x}, d_{y y}$ and $d_{z z}$ are located, respectively, at the $u, v$ and $w$ points. The
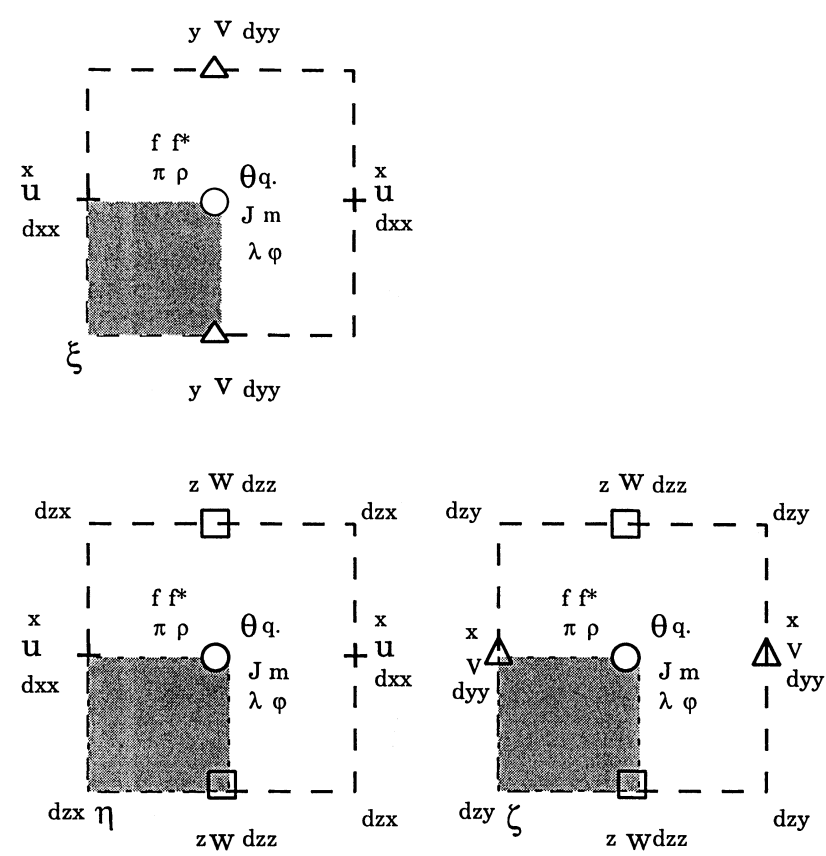

Fig. 3. The discretization on the horizontal (top) and the vertical (bottom). The four points at the corners of each shaded square will share the same values of the indices $i, j$ or $k$
J. P. Lafore et al.: The Meso-NH Atmospheric Simulation System

Jacobian $J$ is located on the mass points. The orography $z_{s}(i, j)$ is defined at the lowest $w$ point.

The discretization is based on second-order finite differences and two-point averages. We adopt the traditional notations of Schuman: $\delta_{x} \alpha, \delta_{y} \alpha$ and $\delta_{z} \alpha$ for finite differences in the directions $\bar{x}, \bar{y}$ and $\bar{z} ; \bar{\alpha}^{x}, \bar{\alpha}^{y}$ and $\bar{\alpha}^{z}$ for averaging in the same directions. For instance, $\delta_{x} \alpha(i, j, k)=(\alpha(i+1, j, k)-\alpha(i, j, k))$ or $(\alpha(i, j, k)$ $-\alpha(i-1, j, k))$, depending of the location of the variable $\alpha, \bar{\alpha}^{x}(i, j, k)=(\alpha(i+1, j, k)+\alpha(i, j, k)) / 2$ or $(\alpha(i, j, k)$ $+\alpha(i-1, j, k)) / 2$, and similarly for the other operators. We found the results quite sensitive to the discretization and location of the metric coefficients and of the Jacobian. The following formulations are currently used

$$
\begin{aligned}
z & =z_{s}+\hat{z}\left(1-\frac{z_{s}}{H}\right), \\
\mathrm{d}_{x x} & ={\left.\overline{\left[\frac{\left(a+\delta_{1} \delta_{2} z\right)}{a}\right.} \frac{1}{m} \delta_{x} \hat{x}\right]}^{x},
\end{aligned}
$$

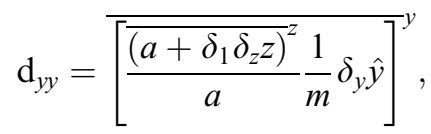

$\mathrm{d}_{z x}=\delta_{x} z$

$\mathrm{d}_{z y}=\delta_{y} z$

$\mathrm{d}_{z z}={\overline{\delta_{z} z}}^{z}$

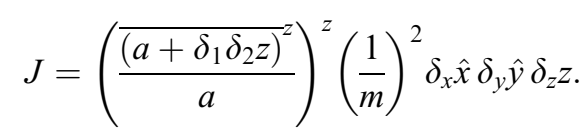

The continuity equations keeps the very simple form

$\delta_{x}\left[\overline{\tilde{\rho}}^{x} U^{c}\right]+\delta_{y}\left[\overline{\tilde{\rho}}^{y} V^{c}\right]+\delta_{z}\left[\overline{\tilde{\rho}}^{z} W^{c}\right]=0$.

The contravariant velocity components read

$$
\overline{\tilde{\rho}}^{x} U^{c}=\overline{\tilde{\rho}}^{x} \frac{u}{\mathrm{~d}_{x x}}
$$

$$
\overline{\tilde{\rho}}^{y} V^{c}=\overline{\tilde{\rho}}^{y} \frac{v}{\mathrm{~d}_{y y}},
$$

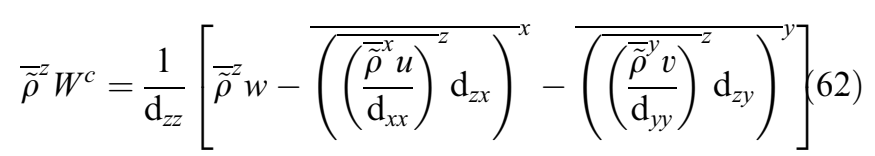

The discretization of the advection and Coriolis terms follows Clark (1977); we do not recall these equations here. The discretization in time is purely explicit, and based on the leapfrog scheme. An Asselin time-filter is also used. 


\subsection{Positive definite advection scheme}

The model offers a variety of advection schemes, from the most simple second-order, centred scheme to the sophisticated MPDATA scheme of Smolarkiewicz and Grabowski (1990). We will hereafter describe a simple Multidimensional Positive Definite Centred Difference Scheme (MPDCD) recently developed, that appears to provide a particularly interesting quality-cost ratio. This applies to the scalar quantities located at the mass points. The generic form of the discretized equation for the scalar quantities is written

$\frac{\partial}{\partial t} \tilde{\rho} \psi=-\sum_{I=1}^{3} \partial_{x I}\left(\tilde{\rho} U_{I}^{c} \psi\right)$.

We define the advective flux of $\psi$ as $F_{I}=\tilde{\rho} U_{I}^{c} \psi$. The solution of Eq. (63) using three time-levels (leapfrog scheme) becomes

$\frac{\tilde{\rho} \psi^{t+\Delta t}}{2 \Delta t}=\frac{\tilde{\rho} \psi^{t-\Delta t}}{2 \Delta t}-\sum_{I=1}^{3} \partial_{x I} F_{I}^{t}$,

where $2 \Delta t$ is the leapfrog time-step. The spatial discretization of the advective part may produce negative values if the sum of the fluxes at the cell faces of a given elementary grid box removes more mass than the mass contained in the grid box. In order to obtain a positive definite advection scheme, an upper limit for the advective flux is applied, following the flux-corrected transport theory of Zalesak (1979). The development of the expression of the limiter factor closely follows the approach of Smolarkiewicz (1989). Assuming that the Courant number is smaller than 1 , the scalar variable computed at $t+\Delta t$ must satisfy the condition

$\psi^{t+\Delta t}=\frac{2 \Delta t}{\tilde{\rho}}\left(F^{I N}-F^{O U T}\right)+\psi^{t-\Delta t}>0$,

where $F^{O U T}$ and $F^{I N}$ are, respectively, the total outgoing and incoming flux summed on all faces of each elementary grid cell (remember that in our coordinate system, the volume of any grid cell is unity in the transformed space). In Eq. (65), the difference $F^{I N}-F^{O U T}$ is equivalent to the total flux in all the directions of the grid cell. A sufficient condition for Eq. (65) is

$\psi^{t-\Delta t} \geq \frac{2 \Delta t}{\tilde{\rho}} F^{O U T}$.

The physical explanation of Eq. (66) is that the outgoing flux $F^{O U T}$ cannot remove more mass in one time-step $(2 \Delta t)$ than that contained in the elementary box at the previous time-step $\left(\psi^{t-\Delta t}\right)$. Equation (66) can be rewritten in the form of a flux limiter ratio $\left(\beta^{O U T}\right)$

$\beta^{O U T}=\frac{\psi^{t-\Delta t}}{\frac{2 \Delta t}{\tilde{\rho}} F^{O U T}}$.

If $\beta^{O U T} \geq 1$, the flux does not need to be modified; if $\beta^{\text {OUT }}<1$, the outgoing flux is overestimated and therefore the flux limiter ratio must be applied. The application of the flux limiter to the flux can be written in a compact manner
$F_{I}=\min \left(1, \beta_{i-1}^{O U T}\left[F_{I}\right]^{+}+\min \left(1, \beta_{i}^{O U T}\right)\left[F_{I}\right]^{-}\right.$,

with $[\cdot]^{+} \equiv \max (0,$.$) and [\cdot]^{-} \equiv \min (0,.) . F_{I}$ is the flux in the face separating the grid cell $i-1$ and $i$ in the $I$ direction. If the flux is positive, it will be corrected by the limiter $\beta_{i-1}^{O U T}$ (of the cell $i-1$ ). If the flux is negative, it will be corrected by the limiter $\beta_{i}^{\text {OUT }}$.

In order to demonstrate the efficiency of this simple scheme, we present a comparison of results obtained with a MPDATA scheme. This test considers the transport of puff of scalar in a flow field over a sharp hill. The flow is characterized by a Froude number defined as $U / N L$ (where $U$ is the mean velocity of the flow, $N$ is the Brunt-väisälä frequency and $L$ is the length of the hill). In our test the Froude number is 10, and the flow is therefore nearly potential. This test is carried out to validate the vertical grid transformation over the mountain and is run in 2D for the sake of simplicity. The stationary flow is obtained by integration of the MesoNH model. Figure 4 shows the vertical velocity and the potential temperature. The initial maximum concentration of the puff is 10 , located at the altitude of the hill top $(200 \mathrm{~m})$. The initial puff diameter is $400 \mathrm{~m}$ both in horizontal and vertical directions. An isotropic grid of 50 $\mathrm{m}$ is used and the Courant number is 0.2 . Figure 5 shows the initial distribution of the puff, and the results corresponding to two puff positions, just above the hill and after the hill passage when the puff should have recovered its initial shape. The results are given for the scheme presented [MPDCD (second-order), for a fourthorder version of the same scheme, and for two versions for the MPDATA scheme (2 and 3 iterations)]. The cost of MPDCD at second order is about 3.4 times more expensive than the non-corrected second-order scheme, but two and three times less expensive than the two versions of MPDATA, respectively.

Other tests have been performed, in particular using the deformational flow field of Smolarkiewicz (1982), for which the analytical solution is supplied by Staniforth et al. (1987). Again, MPDCD performed nearly as well as MPDATA, at a lower cost.

\section{The pressure solver}

A distinctive feature of the anelastic system of equations is the requirement of solving an elliptic pressure equation with high accuracy. This is a difficult point for many other non-hydrostatic models, and so we shall discuss it in some detail.

\subsection{Formulation of the elliptic problem}

We seek the discretized form of Eq.(16). We shall denote by $G D I V$ the discretized divergence operator. Furthermore, in order to avoid the error growth on the wind divergence, we adopt the widely used method of replacing $\vec{S}$ by $\vec{B}=\vec{S}-\tilde{\rho} \vec{U}(t-\Delta t) / 2 \Delta t \quad$ (Williams, 1969). As already explained, the divergence operator can be expressed in terms of the contravariant compo- 

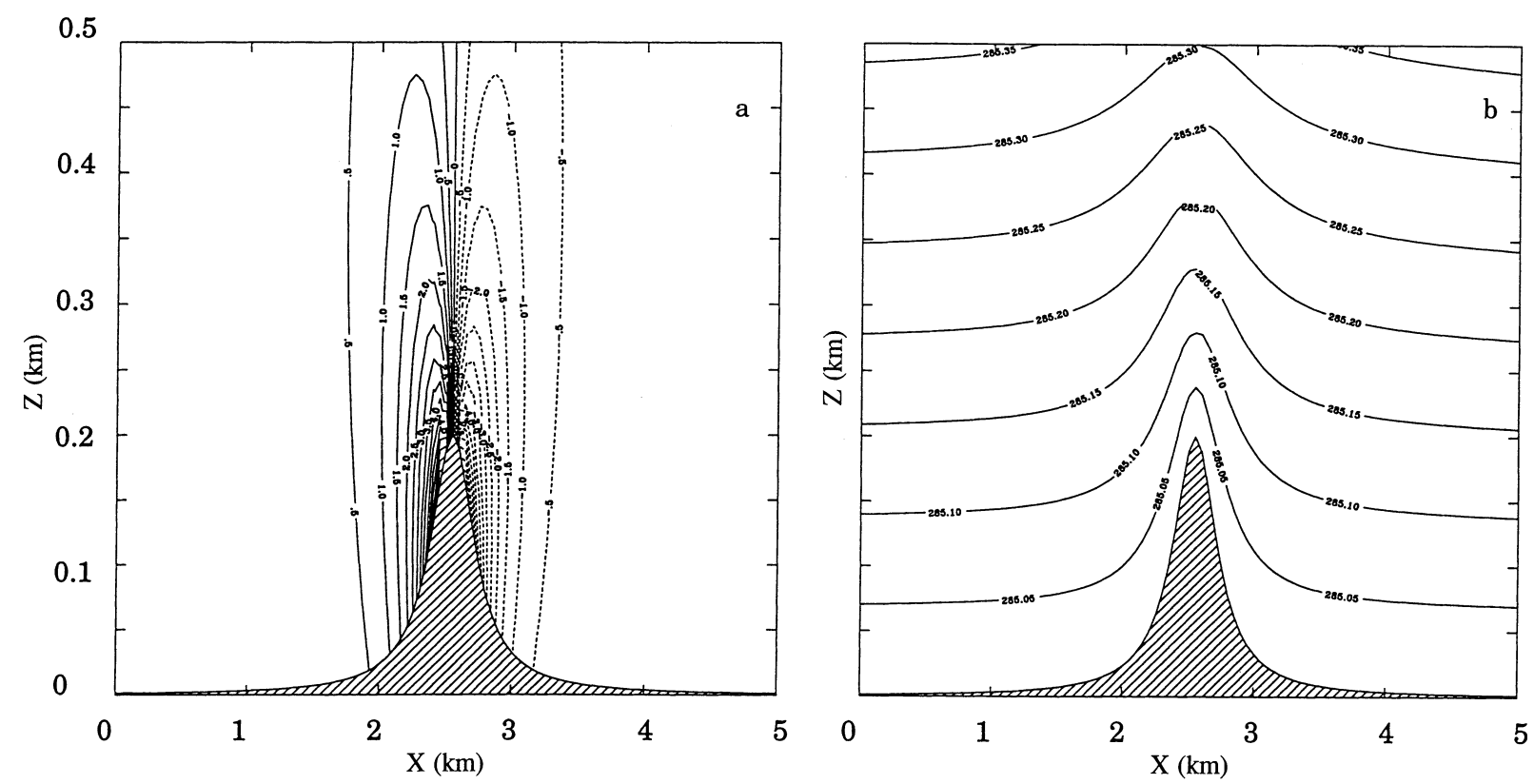

Fig. 4a,b. Solution obtained by the model for the nearly potential flow field used in the test of the advection scheme. a Vertical velocity, contour interval $0.5 \mathrm{~m} \mathrm{~s}^{-1}$; b potential temperature, contour interval $0.05 \mathrm{~K}$. The coordinate lines are labelled in $\mathrm{km}$

nents of a vector. The discrete form of the contravariant components of $\vec{B}$ is

$B^{c 1}=\frac{B_{1}}{\mathrm{~d}_{x x}}$,

$B^{c 2}=\frac{B_{2}}{\mathrm{~d}_{y y}}$,

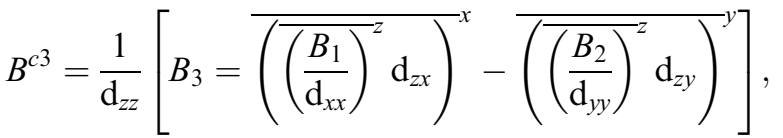

where $\left(B_{1}, B_{2}, B_{3}\right)$ are the Cartesian components of $\vec{B}$. We then have

$G D I V(\vec{B})=\delta_{x} B^{c 1}+\delta_{y} B^{c 2}+\delta_{z} B^{c 3}$

The left-hand side of Eq. (16) is discretized in a similar way, resulting in

$$
\begin{aligned}
& Q(\Phi)=\operatorname{GDIV}(\tilde{\rho} \vec{\nabla} \Phi)=\delta_{x}\left[\overline{\tilde{\rho}}^{x} \frac{\delta_{x} \Phi}{\mathrm{d}_{x x}^{2}}\right]+\delta_{y}\left[\overline{\tilde{\rho}}^{y} \frac{\delta_{y} \Phi}{\mathrm{d}_{y y}^{2}}\right] \\
& +\delta_{z}\left[\overline{\tilde{\rho}}^{z} \frac{\delta_{z} \Phi}{\mathrm{d}_{z z}^{2}}\right]-\delta_{x}\left[\overline{\tilde{\rho}}^{x} \frac{1}{\mathrm{~d}_{x x}^{2}} \overline{\left(\mathrm{d}_{z x} \overline{\left(\frac{\delta_{z} \Phi}{\mathrm{d}_{z z}}\right)^{x}}\right)^{x}}\right] \\
& -\delta_{y}\left[\overline{\tilde{\rho}}^{y} \frac{1}{\mathrm{~d}_{y y}^{2}} \overline{\left(\mathrm{d}_{z y} \overline{\left(\frac{\delta_{z} \Phi}{\mathrm{d}_{z z}}\right)^{y}}\right)^{2}}\right]
\end{aligned}
$$

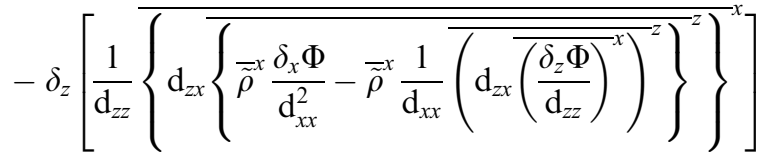

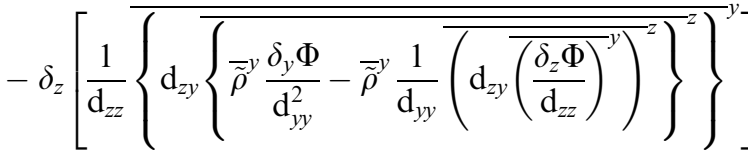

The elliptic equation is completed by boundary conditions. As explained, there are many different formulations for the boundary conditions. However, all of them may be expressed as a time tendency for the normal component of the wind at the boundary (except in the special case of cyclic boundary conditions). From Eq. (15), we can then infer the normal component of the pressure gradient when the source components $\vec{S} \cdot \vec{n}$ are known. The result is a non-homogeneous Neumann condition for the full elliptic problem

$\frac{\partial \phi}{\partial n}=\vec{S} \cdot \vec{n}-\frac{\partial}{\partial t}\left(\rho_{d r e f} \vec{U} \cdot \vec{n}\right)$.

We render this condition homogeneous by following the procedure of Williams (1969), also used recently by Xue and Thorpe (1991). The pressure function is replaced by $\phi^{\prime}=\phi-\phi_{0}$, where $\phi_{0}$ is zero inside the computational domain and non-zero at the outer points, so that the 1.b.c. of Eq. (74) is satisfied. Now $\phi^{\prime}$ satisfies the elliptic problem:

$$
\begin{aligned}
\frac{\partial \phi^{\prime}}{\partial n} & =0 & & \text { at the boundary, } \\
Q\left(\phi^{\prime}\right) & =\operatorname{GDIV}\left(\vec{S}^{\prime}\right) & & \text { at points just inside the boundary, } \\
Q\left(\phi^{\prime}\right) & =\operatorname{GDIV}(\vec{S}) & & \text { at the other points, }
\end{aligned}
$$

where $S^{\prime}$ is obtained by replacing components $S_{x}$ and $S_{y}$ by $\frac{\partial\left(\rho_{\text {dref }} u\right)}{\partial t}$ and $\frac{\partial\left(\rho_{\text {dref }} v\right)}{\partial t}$, respectively. This method is not used when the cyclic boundary conditions are selected.

\subsection{Preconditioning technique}

In order to solve efficiently the preceding problem, we use the preconditioning technique developed by Ber- 

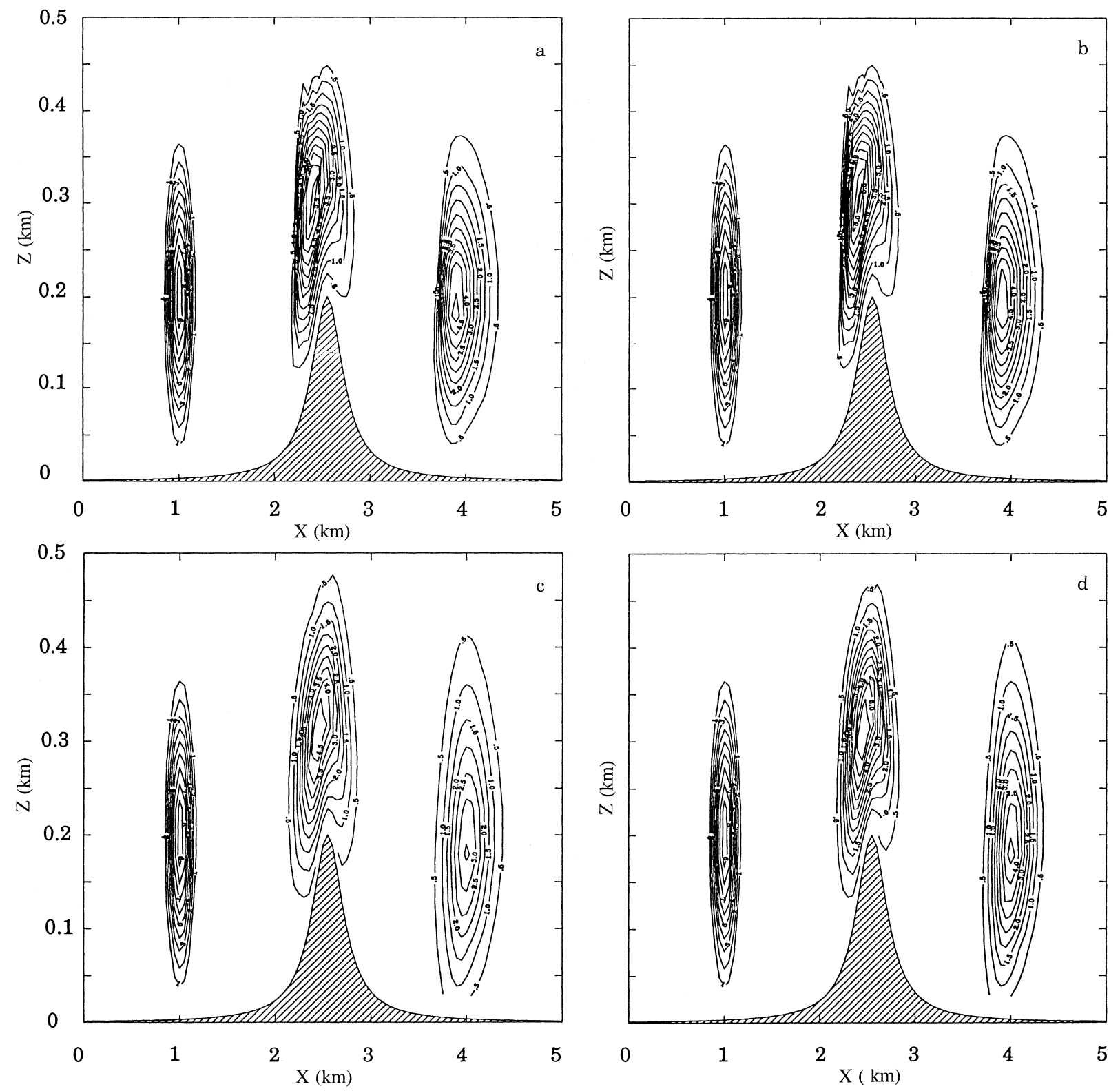

Fig. 5a-d. Distribution of the scalar puff at $t=0, t=150=$ and $t=300$ using several positive definite advection schemes: a MPDCD (secondorder); b MPDCD (fourth-order); c MPDATA ( 2 iter.); d MPDATA ( 3 iter.). The initial scalar puff maximum is 10 , and the contour interval is 0.5

nardet (1995) which makes use of the solution of the "flat" problem. "Flat" means that the topography and the earth's curvature are neglected. It follows that $d_{z x}$ and $d_{z y}$ vanish, and only the first three terms of Eq. (73) remain. Since $\tilde{\rho}$ only depends on $z$, the flat operator $F$ can be rewritten as

$$
\begin{aligned}
& F \Phi=\langle\tilde{\rho}\rangle_{x, y}\left[\frac{1}{\left\langle\mathrm{~d}_{x x}^{2}\right\rangle_{x y}} \delta_{x^{2}} \Phi+\frac{1}{\left\langle\mathrm{~d}_{y y}^{2}\right\rangle_{x y}} \delta_{y^{2}} \Phi\right] \\
& +\delta \overline{\langle\tilde{\rho}\rangle_{x, y}} z \frac{1}{\left\langle\mathrm{~d}_{z z}^{2}\right\rangle_{x y}} \delta_{z} \Phi .
\end{aligned}
$$

The method then consists in treating the horizontal part of $F$ in the Fourier space. First, we compute the FFT of the right-hand side of the equation. Then we introduce the horizontal Fourier decomposition of $F$ completed by its classical vertical part. This results in $i \max \times i \max$ tridiagonal matrices where every matrix corresponds to a different horizontal mode. A classical tridiagonal matrix inversion is performed for each horizontal mode, which allows us to compute the solution in the Fourier space. Finally, we apply an inverse FFT to obtain $\Phi$ in the physical space.

\subsection{Richardson's method}

Then, we developed two different iterative methods [the conjugate gradient method after Kapitza and Eppel (1992) and the Richardson method, after Golub and Meurant (1983)]. For both methods, the first guess is 
also set equal to the solution of the flat problem. The conjugate gradient method will not be discussed here as it has been found to be less efficient. We write the problem as

$F^{-1} Q x=F^{-1} y$,

where $x$ is the unknown, $y$ is the right-hand side, $Q$ is the complete operator defined in Eq. (73) and $F$ is the flat operator, used as a preconditioning matrix.

The classical Richardson iterative method reads

$x^{(n+1)}=x^{(n)}+\alpha\left(F^{-1} y-F^{-1} Q x^{(n)}\right)$,

where $x^{(n)}$ is the $n$-th iteration of $x$. This method includes a relaxation factor $\alpha$, allowing for an under-relaxation which is useful to improve the solver convergence in the presence of large slopes.

The efficiency of the pressure solver is checked by evaluating the order of magnitude of the residual velocity divergence, after a given number of iterations. An example is shown on Fig. 6. In the usual practice, four iterations are considered to be sufficient. This number may need to be increased when very large slopes are present in the domain. The cost of the solver is currently $1.610^{-4} \mathrm{~s} \mathrm{Cpu}$ per grid point and per iteration on a single processor of a Cray C90 machine. The conjugate gradient method, also available, has a wider range of applications, but at approximately double the cost.

\section{Examples of idealized validation simulations}

We provide here several basic examples which are used for the purpose of validation of the adiabatic part of the model. These simulations are performed after any major change in the model code, or after the model installation on a new site, in order to check the good functioning of the code. This is introduced by a short discussion on time-step selection.

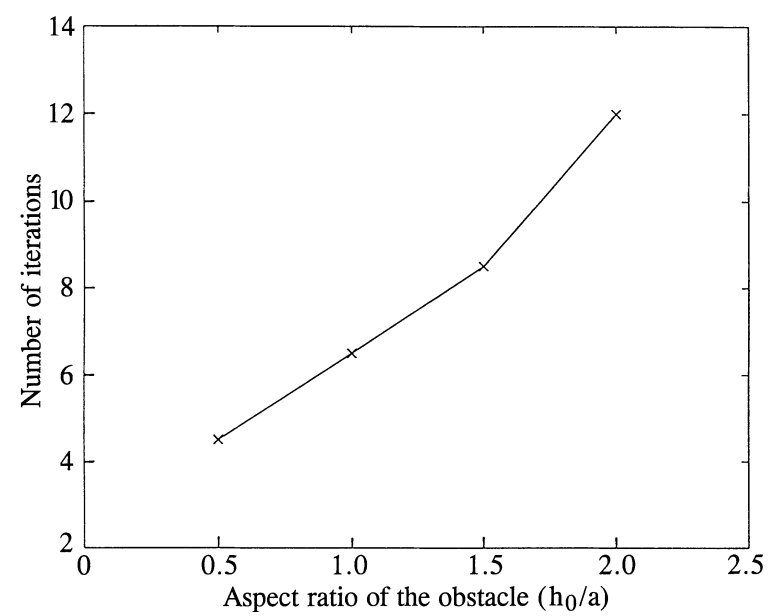

Fig. 6. A measure of the solver performance. The case-study is a simulation of 2D mountain waves. The number of solver iterations needed to achieve a divergence of $10^{-10} \mathrm{~s}^{-1}$ is shown as function of the mountain aspect ratio

\subsection{Stability requirements}

A stability analysis is based on the two-dimensional Boussineq equations linearized around a hydrostatic basic state. Fourier decompositions are then used to account for the effect of the discretizations (explicit in time) in the previous equations. As this system of discrete equations admits solutions only when its determinant vanishes, this condition results in a discrete dispersion relationship for internal waves. Solving this relation leads to a criterion on the limit of time-step, and the most restrictive form can be expressed as

$\Delta t_{\lim }=\left(\frac{U}{\Delta x}+\frac{N|\cos (\pi \Delta z / 2 H)|}{\left[1+\left(\frac{\Delta x}{\Delta z} \sin (\pi \Delta z / 2 H)\right)^{2}\right]^{1 / 2}}\right)^{-1}$,

where $\Delta t_{\text {lim }}$ is the limit of the time-step for stable integration, $H$ is the height of the model top, $\Delta x$ and $\Delta z$ are, respectively, the horizontal and vertical grid intervals, $U$ is the mean flow speed and $N$ is the Brunt-Vaiisälä frequency. The dependence of $\log \left(\Delta t_{\mathrm{lim}}\right)$, with respect to $\Delta x$ and $H$ is shown in Fig. 7. We may note the following points: due to the anelastic hypothesis and to the rigid lid at the top of the model, fast-travelling waves such as external gravity waves or acoustic waves are filtered out and do not appear in the expression of the time-step limitation, which allows rather large time-steps, even with our time-explicit scheme. At small grid aspect ratio $\Delta x / \Delta z, \Delta t_{\text {lim }}$ is quasi-independent of the domain height $H$. This will be the case in general for meso-gamma scales. For larger grid aspect ratio $\Delta x / \Delta z, \Delta t_{\text {lim }}$ depends on $H$ (meso- $\alpha$ and- $\beta$ scales). At these scales, it is beneficial to reduce $H$ in order to increase the time-step, inasmuch as the physical problem of interest allows it.

This method of determining the limit of time-step must be used with care, because the basic equations are

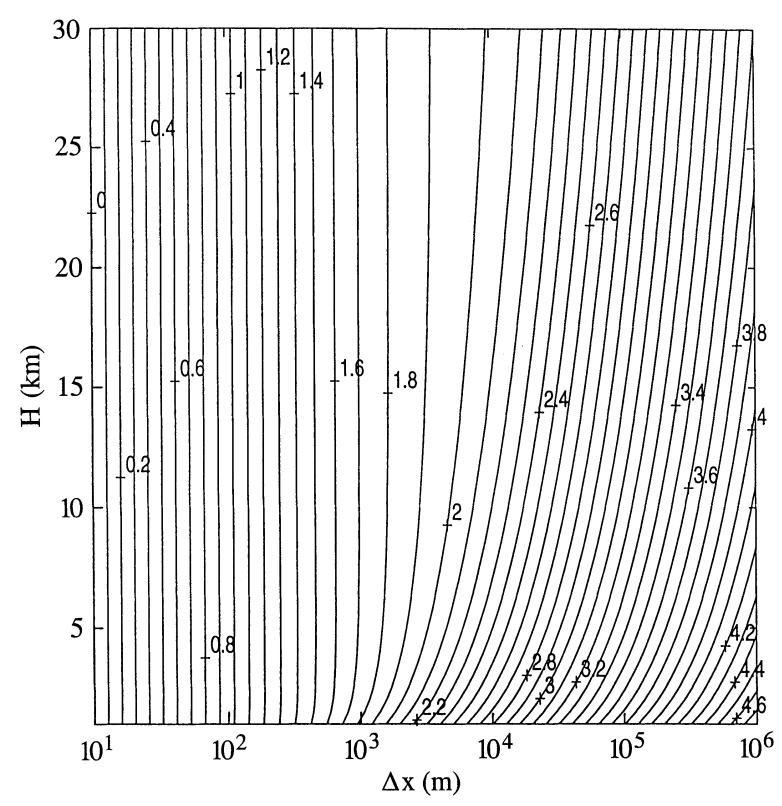

Fig. 7. Values of $\log \left(\Delta t_{\lim }\right)$, where $\Delta t_{\lim }$ is in seconds, for $U=10 \mathrm{~m} \mathrm{~s}^{-1}, N=0.01 \mathrm{~s}^{-1}$, and $\Delta z=250 \mathrm{~m}$ 
largely simplified in comparison with the Meso-NH equations. In practice, it has been verified on several examples that an effective method to determine the timestep limit is to apply a security coefficient of 0.8 to Eq. (78). Note, however, that although the stability analysis carried out in two dimensions only, the result seems to apply to 3D cases as well (with a correction factor of $\sqrt{2}$ when $\Delta x=\Delta y$, or with the most restrictive condition between $x$ and $y$ if the grid is very anisotropic). In the normal use of the model including the physical package, other constraints may of course limits the time-step. Also, the vertical velocity may become large if the model resolves strong convective drafts, and this will place an additional restriction involving the vertical grid size.

\subsection{Idealized vortices from aircraft wake}

We start with an example of very small scale flow, which is currently the object of intensive research: the system of two vortices left behind by an aircraft at take-off or landing. It is well known that each vortex induces a negative vertical velocity on the other. As the pair approaches the ground, the distance between the two vortices increases, as a result of the boundary condition. Recent research has shown that a secondary vortex is generated close to the ground, inducing a rebound. This is due to the no-slip condition in real, viscous flow, and has also been simulated by our model. However, we will only present here the inviscid case, where the flow is potential, and the trajectory is given by the most simple equation (see e.g. Saffman, 1979)

$\frac{1}{x^{2}}+\frac{1}{z^{2}}=C$ ste.

The case has been simulated with the model with the conditions given in Table 1. The computed trajectory for the right-hand vortex is shown in Fig. 8, together with the analytic solution. In this case, the vortices had a maximum tangential velocity of about $10 \mathrm{~m} \mathrm{~s}^{-1}$ and an extension of few metres. They were released at $65 \mathrm{~m}$ above the ground, and finished their trajectory by leaving the computation domain (via the open boundary conditions) at a height of $14 \mathrm{~m}$ above the ground. This took about $1 \mathrm{~min}$.

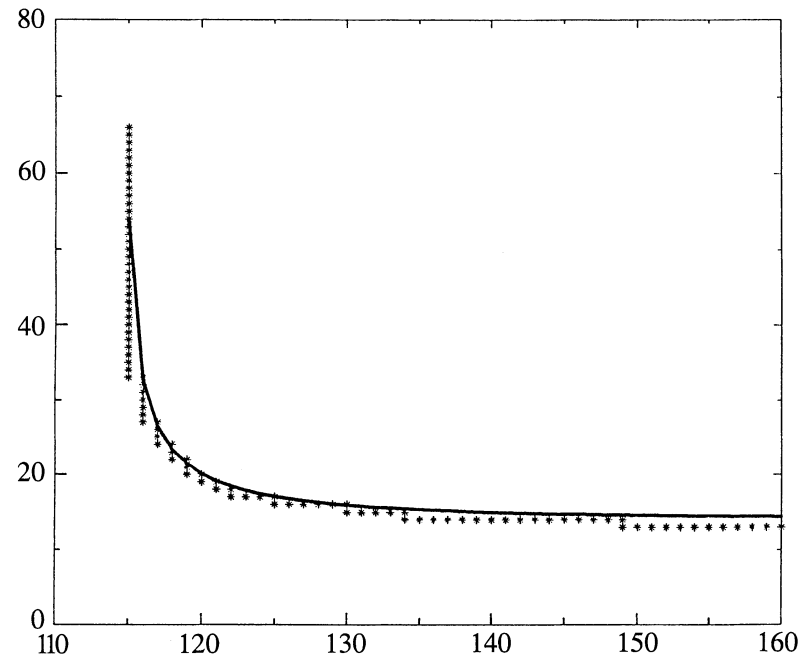

Fig. 8. Result of the control simulation for aircraft wake vortices. Comparison of the right-hand vortex trajectory simulated by the model (stars) with the analytical solution [continuous line from Eq.(78)]. The coordinate lines are labelled in $\mathrm{m}$, and the pair or vortices is initially released at the height of $65 \mathrm{~m}$

\subsection{Idealized mountain waves}

We will next present several cases of mountain waves at the meso-beta and gamma scales. First, three cases of 2D mountain-waves are discussed. The mountain of half-width $a$ is given by

$h(x)=h_{\max } /\left(1+(x / a)^{2}\right)$.

The upstream flow is vertically homogeneous, with a static stability of $N=0.01 \mathrm{~s}^{-1}$ and wind speed $U=10 \mathrm{~ms}^{-1}$. For these simulations, cyclic lateral boundary conditions are assumed but a narrow sponge zone is defined near the lateral boundary to damp outwardsradiating waves. The perturbation is fully resolved on the lowest 25 model levels only, as the upper layers are devoted to a sine-square shaped absorbing layer with a maximal damping rate of $5 U / a=0.005 \mathrm{~s}^{-1}$.

For the first case, the flow is linear with $h_{\max }=10 \mathrm{~m}$, and hydrostatic with $a=5 \Delta x=10 \mathrm{~km}$. The model is integrated up to the non-dimensional time $t^{*}=U t / a=60$. The model is run without explicit numerical diffusion. The results are shown in Fig. 9.

Table 1. Some characteristics of the control simulations. The CPU time is measured on a single Cray C90 processor

\begin{tabular}{|c|c|c|c|c|c|c|c|}
\hline case & configuration & $\Delta x, \Delta z$ & $\Delta t$ & dimensions & duration & memory & CPU \\
\hline aircraft vortices & open LBC & $1 \mathrm{~m}, \quad 1 \mathrm{~m}$ & $0.06 \mathrm{~s}$ & 200,100 & $60 \mathrm{~s}$ & $7 \mathrm{Mw}$ & $600 \mathrm{~s}$ \\
\hline $\begin{array}{l}\text { mountain waves } \\
\text { (hydrostatic) }\end{array}$ & $\begin{array}{l}\text { cyclic LBC } \\
+ \text { sponge }\end{array}$ & $2 \mathrm{~km}, 250 \mathrm{~m}$ & $20 \mathrm{~s}$ & 90,63 & $60000 \mathrm{~s}$ & $2.4 \mathrm{Mw}$ & $167 \mathrm{~s}$ \\
\hline $\begin{array}{l}\text { mountain waves } \\
\text { (non-hydrostatic) }\end{array}$ & idem & $133 \mathrm{~m}, \quad 250 \mathrm{~m}$ & $2 \mathrm{~s}$ & 180,63 & $4000 \mathrm{~s}$ & $3.3 \mathrm{Mw}$ & $200 \mathrm{~s}$ \\
\hline $\begin{array}{l}\text { mountain waves } \\
\text { (3D) }\end{array}$ & $\begin{array}{l}\text { cyclic LBC } \\
+ \text { sponge }\end{array}$ & $2 \mathrm{~km}, 100 \mathrm{~m}$ & $45 \mathrm{~s}$ & $128,96,180$ & $40000 \mathrm{~s}$ & $110 \mathrm{Mw}$ & $7400 \mathrm{~s}$ \\
\hline baroclinic waves & $\begin{array}{l}\text { cyclic LBC } \\
+ \text { wall }\end{array}$ & $85 \mathrm{~km}, 500 \mathrm{~m}$ & $200 \mathrm{~s}$ & $96,48,33$ & 10 days & $8.5 \mathrm{Mw}$ & $1886 \mathrm{~s}$ \\
\hline
\end{tabular}


They closely resemble the analytical solution. Furthermore, the associated momentum flux attains $95 \%$ of the theoretical linear solution, and the computed pressure drag attains $96.5 \%$ of the theoretical value.

In the next case, a non-linear flow has been simulated with a mountain height of $h_{\max }=500 \mathrm{~m}$. The horizontal fourth-order numerical diffusion is applied with a damping time-scale of $75 \mathrm{~s}$ for the $2 \Delta x$ waves. The model results at $t^{*}=40$ are shown in Fig. 10. The wave flux estimates indicate that there is a significant nonlinear amplification, as expected from the theory.

The following simulation illustrates a linear, nonhydrostatic case. The model solution in Fig. 11 shows the slant-wise propagation of the non-hydrostatic wave until it reaches the domain boundary at $t^{*}=30$. The computed pressure drag takes a value of $28 \%$ of the linear hydrostatic result, which is in excellent agreement with the theoretical solution. The wave momentum flux also has a correct value.

As a final test on mountain waves, we consider the less classical problem of the 3D flow around a bellshaped mountain defined by

$$
h_{s}(x, y)=\frac{h}{\left[1+\left(\frac{x^{2}}{L_{x}^{2}}\right)+\left(\frac{y^{2}}{L_{y}^{2}}\right)\right]^{\frac{3}{2}}} \text {. }
$$

The mountain characteristics are defined as $h=50 \mathrm{~m}$, $L_{x}=L_{y}=10 \mathrm{~km}$. The upstream values of wind and temperature are $10 \mathrm{~m} \mathrm{~s}^{-1}$ and $285 \mathrm{~K}$. Thus, the flow is quasi-hydrostatic and quasi-linear, and may be compared to existing analytical solutions (e.g. Smith, 1980). The cyclic lateral boundary conditions are used, and near the lateral boundaries, relaxation towards the initial state is applied on 16 points along $x$ and on three points along $y$, with a damping rate of $0.0016 \mathrm{~s}^{-1}$. A sponge zone extending from $12570 \mathrm{~m}$ to the top of the

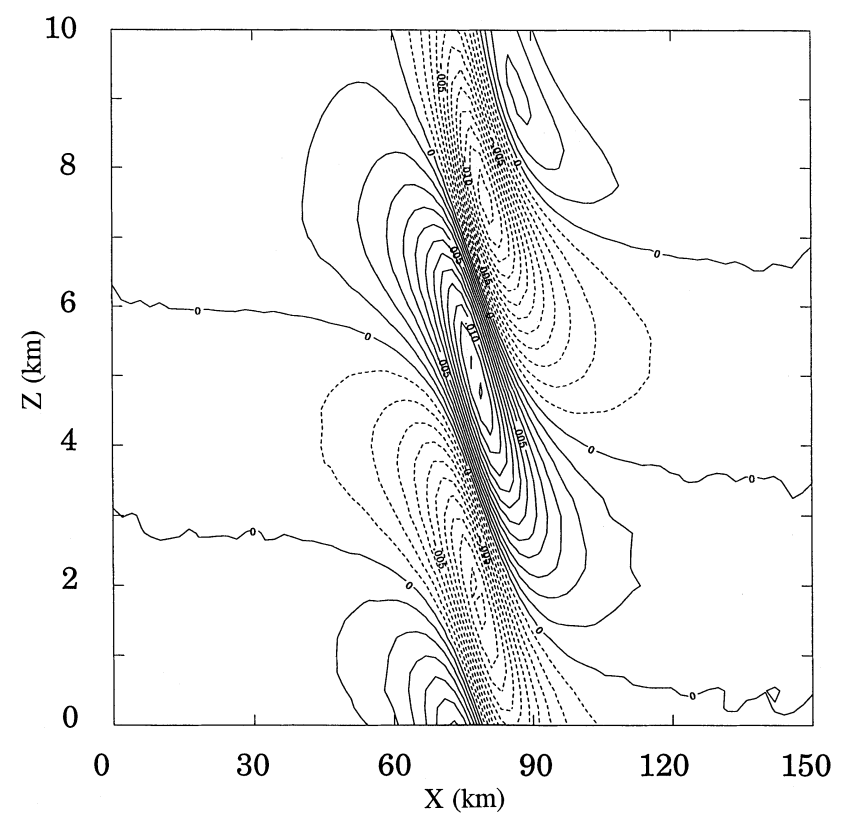

Fig. 9. Field of vertical velocity in the control simulation, for $2 \mathrm{D}$ linear, hydrostatic mountains waves; contour interval is $0.001 \mathrm{~m} \mathrm{~s}^{-1}$

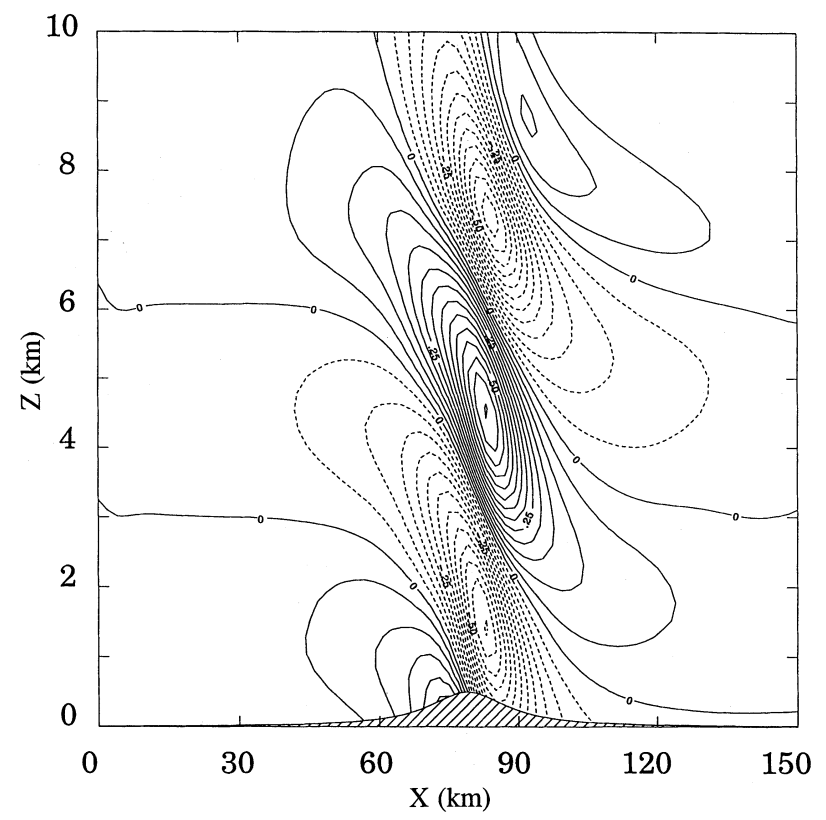

Fig. 10. Field of vertical velocity in the control simulation, for $2 \mathrm{D}$ non-linear, hydrostatic mountain waves; contour interval is $0.05 \mathrm{~m} \mathrm{~s}^{-1}$

model is used to prevent reflexion of gravity waves. The integration is carried out until a non-dimensional time $t^{*}=t U / L_{x} \approx 40$ is reached. This is considered a good approximation of steady state. The $u$ and $v$ components in a horizontal cross-section at $z=250 \mathrm{~m}$ are shown in Fig. 12. A comparison with the analytic solution shows a very good correspondence. The vertical flux of horizontal momentum is vertical, and balances exactly the surface pressure drag. The agreement with the analytical value is better than $90 \%$. More complete results on this case are presented by Héreil (1996).

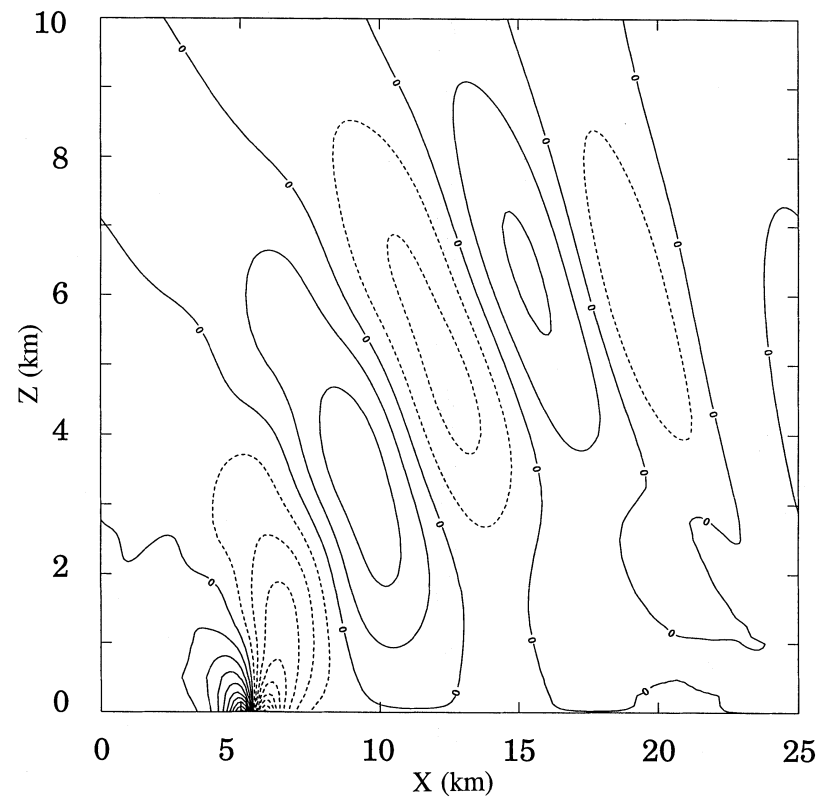

Fig. 11. Field of vertical velocity in the control simulation for $2 \mathrm{D}$ linear, non-hydrostatic mountain waves; contour interval is $0.01 \mathrm{~m} \mathrm{~s}^{-1}$ 

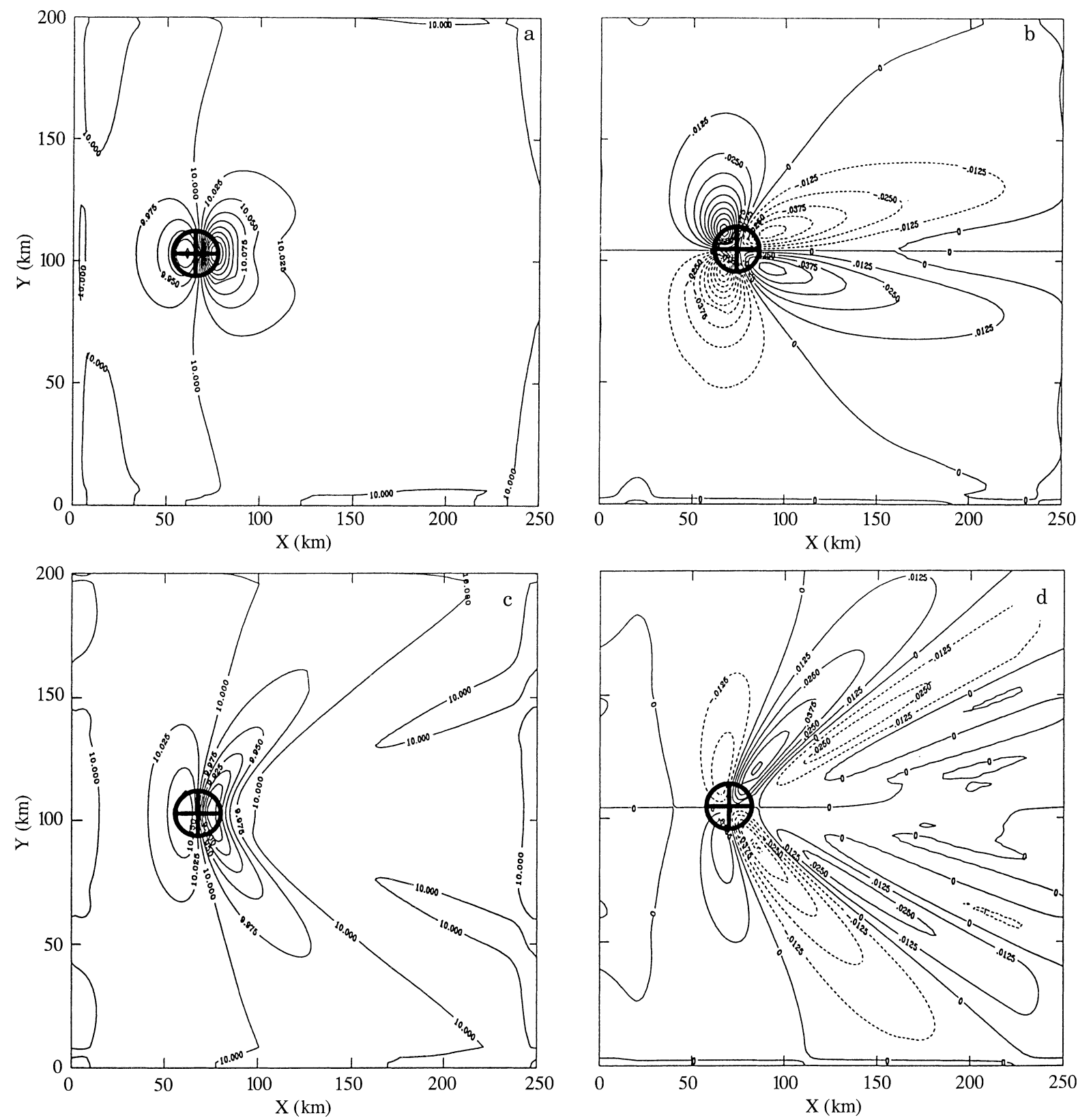

Fig. 12a-d. Results of the control simulation for $3 \mathrm{D}$ linear, hydrostatic mountain waves. The cross shows the position of the mountain. The fields shown are the $u$ component of velocity at a $250 \mathrm{~m}$ and $\mathbf{c} 3000 \mathrm{~m}$ altitude, and the $v$ component of velocity at b $250 \mathrm{~m}$ and $\mathbf{d} 3000 \mathrm{~m}$. Contour intervals are $0.025 \mathrm{~m} \mathrm{~s}^{-1}$ for $u$ and $0.0125 \mathrm{~m} \mathrm{~s}^{-1}$ for $v$

\subsection{Idealized $3 D$ baroclinic waves}

As a third validation case for the model dynamics, we will adress the meso-alpha scale, with a 3D non-linear simulation of a baroclinic-wave life cycle. The 3D nonlinear development of normal-mode type perturbations superimposed on simple zonal jets with meridional and vertical variations has received a great deal of attention in the past. It has been treated with a wide range of model types, and thereby offers a good test-bench for validation studies. For instance, the base state defined by Hoskins and West (1979) with a semi-geostrophic approach, also considered recently by Polavarapu and Peltier (1990) with a non-hydrostatic, anelastic model, by Snyder et al. (1991) with a non-hydrostatic, compressible model and Lalaurette et al. (1994) with a hydrostatic, primitive equation model. Here we use the Meso-NH model to reproduce the Lalaurette et al. (1994) case-study, which was initially performed with the PERIDOT hydrostatic model formerly used at Météo-France. This case features a strong zonal jet within the troposhere and the stratosphere, with a realistic tropopause in between. Both upper-tropospheric and surface frontogenetic processes are present.

The Meso-NH configuration for these tests is dry and nearly adiabatic. No surface friction or Ekman layer is present. The only diabatic term used is a small horizontal diffusion operator in the non-linear simula- 
tions. No explicit horizontal diffusion is used in the quasi-linear runs. In order to allow for exact comparison with the study by Lalaurette, the Meso-NH fourth-order horizontal diffusion scheme is replaced by a more standard second-order scheme. An important point is that this second-order scheme directly operates on the whole model fields (not on the deviations from the LS fields, as the standard scheme described earlier). The edamping time used in $7000 \mathrm{~s}$ for the $2 \Delta x$ wave. We believe the diffusion scheme used for particular study is much more diffusive than the standard Meso-NH scheme. No spherical effect is included, the Cartesian and thin-shell approximations are made. The model domain is periodic in the zonal (west-east) direction, and rigid-wall conditions are set at the northern and southern model boundaries.

Life-cycle simulations of baroclinic waves are classically performed in three stages: first, a base state is defined, next a quasi-linear simulation is carried out to obtain a small-amplitude normal-mode solution, finally a non-linear finite amplitude simulation is carried out using the normal-mode solution as an initial condition.

The zonally uniform base state for the simulations is shown in Fig. 13; the zonal wind field (upper panel) is directly taken from Lalaurette et al. (1994) data, it displays a jet with smooth horizontal and vertical shears in both the troposphere and stratosphere. The maximum jet intensity is $28 \mathrm{~m} \mathrm{~s}^{-1}$ at about $10 \mathrm{~km}$ and the surface wind is zero. The thermal structure (lower panel) is computed to be in thermal wind balance with the jet profile, using the Meso-NH equations. This field is visually identical to the thermal structure provided by the reference paper.

The selection of the most unstable $4000-\mathrm{km}$ wavelength quasi-normal mode is made iteratively, as a suggested by Lalaurette et al. (1994). The model without horizontal diffusion is initialized with the base-state zonal jet modified by a small arbitrary monochromatic zonal perturbation (meridional wind amplitude $1.7 \mathrm{~m} \mathrm{~s}^{-1}$ ), and integrated for 15 days. Every $24 \mathrm{~h}$, the growth of the perturbation is monitored, and its amplitude is rescaled to restore the initial meridional wind amplitude $\left(1.7 \mathrm{~m} \mathrm{~s}^{-1}\right)$. During this iterative loop, the daily growth progressively becomes stationary, and the growth rate reaches a value close to $4.6810^{-6} \mathrm{~s}^{-1}$ at day 15 . The perturbation phase velocity at the same time is about $7.9 \mathrm{~m} \mathrm{~s}^{-1}$. Similar growth rate and phase velocity were found by Lalaurette et al. (1994); $4.410^{-6} \pm 0.310^{-6} \mathrm{~s}^{-1}$ and $8.3 \pm 0.3 \mathrm{~m} \mathrm{~s}^{-1}$ respectively.

The non-linear integration is then run for 10 days. As the wave develops, a warm front appears on the north-eastern side of the cyclone and forms the most active part of the wave during the early stages. Later, a cold front forms to the south-east of the cyclone and bends around the anticyclone. This tendency for warm frontogenesis to occur before cold frontogenesis is a classical character of primitive equation simulations of baroclinic waves in meridionally limited channels. As can be seen in Fig. 14, our results are in fair agreement with Lalaurette et al. (1994). At the surface level (upper
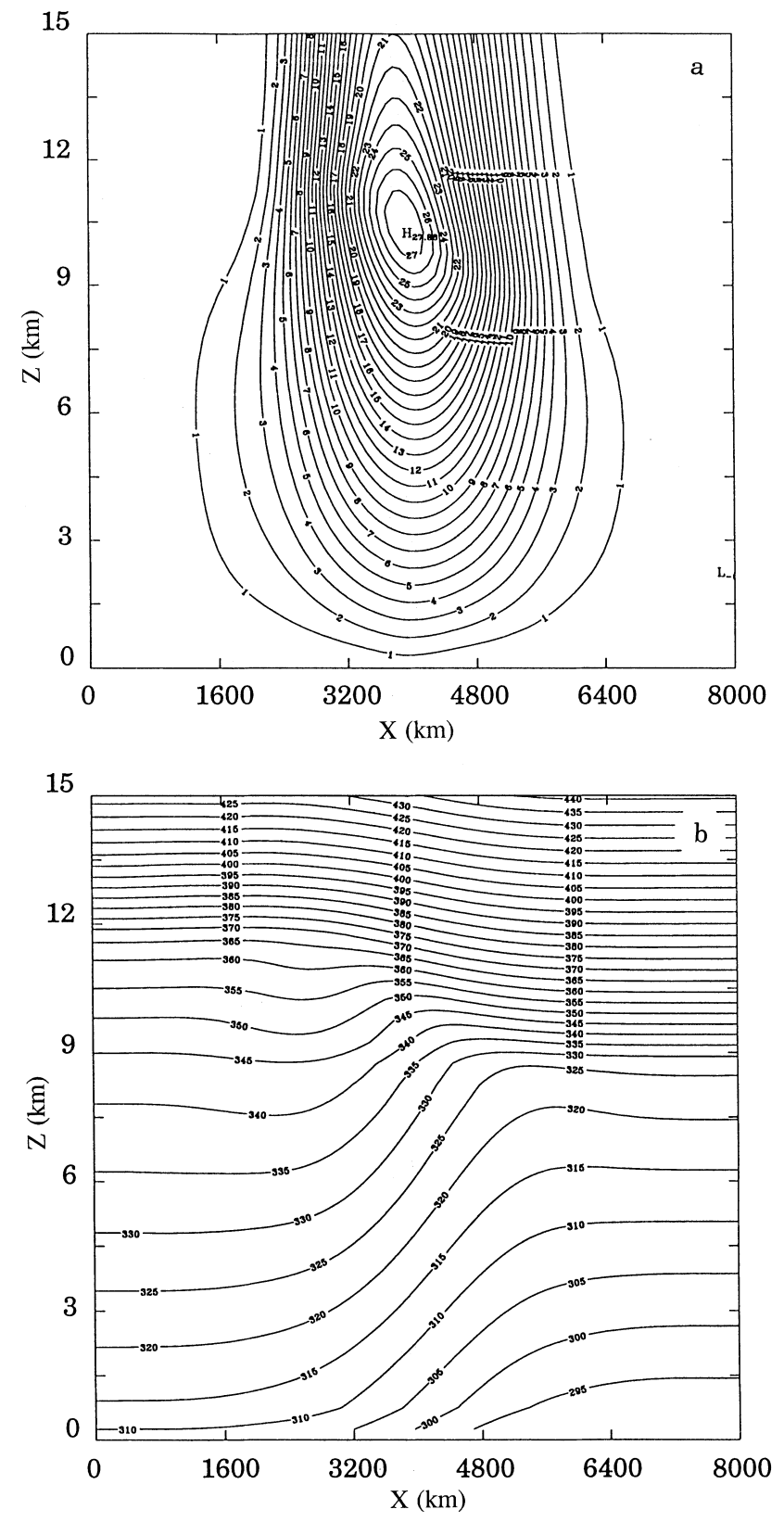

Fig. 13a,b. Basic state used for the baroclinic wave control simulation. a Meridional cross-section of the zonal wind, contour interval $1 \mathrm{~ms}^{-1}$; b potential temperature, contour interval $5 \mathrm{~K}$

panels), both models predict the spiralling of the warm sector towards the cyclone centre, the wave intensity is roughly the same with a deepening of about $35 \mathrm{hPa}$ by day 8 in both cases. The conclusion of this test is that the current formulation of Meso- $\mathrm{NH}$ reproduces the results previously found by other models within acceptable limits of errors. The origin of the remaining discrepancy has not been clearly elucidated at the present time and requires further work. It could be related to the approximation involved in the Lipps and Hemler formulation of the basic equations, or to small differences in the diffusive terms. The on-going work on some more exact formulations may solve this question. 

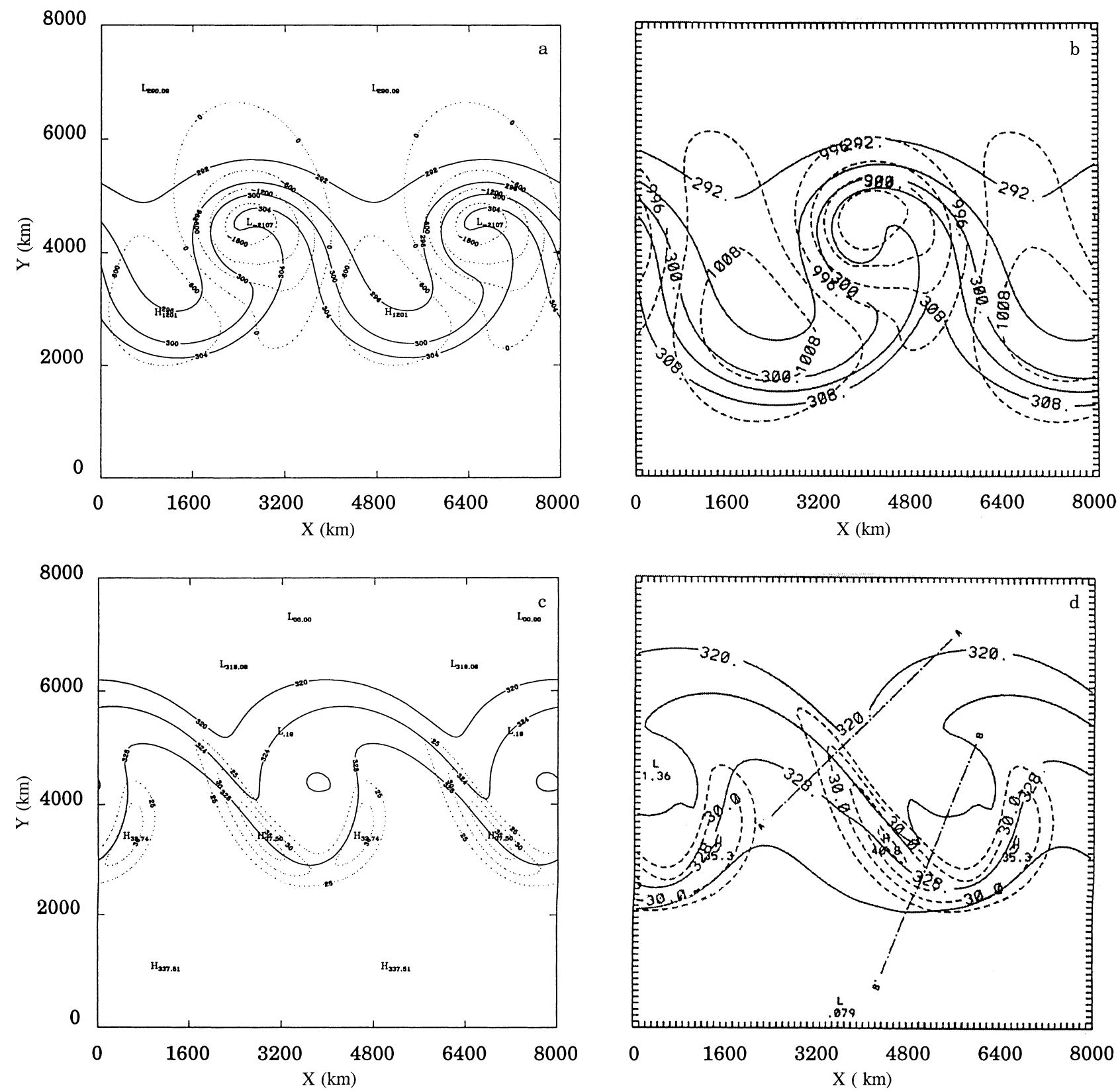

Fig. 14a-d. Results from the baroclinic wave control simulation, compared with those of Lalaurette et al. (1994), using a primitive equation, hydrostatic model, a Surface-level potential temperature (solidlines, $4 \mathrm{~K}$ ) and pseudo-geopotential perturbation $\Phi$ (dashed, $6 \mathrm{hPa}$ ) computed by Meso-NH at day 8; b surface-level potential temperature (solidlines, $4 \mathrm{~K}$ ) and pressure (dashed $6 \mathrm{~h} \mathrm{~Pa}$ )

\section{Other aspects, recent evolution and conclusion}

It is not possible to describe all the available functionalities of the model in a single paper. We will summarize here the important points that had to be left out.

The model has now a fairly complete physical package. The turbulence parametrization is a one-anda-half-order closure scheme, involving a prognostic equation for turbulence kinetic energy, computation of mixing lengths, and a Richardson-number-dependent

computed with the reference model at day 8. c potential temperature at $7000 \mathrm{~m}$ (solid lines $4 \mathrm{~K}$ ) and wind speed at $9000 \mathrm{~m}$ (dashed, $5 \mathrm{~m} \mathrm{~s}^{-1}$ ) computed by Meso-NH at day 10; d potential temperature at $7000 \mathrm{~m}$ (solidlines, $4 \mathrm{~K}$ ) and wind speed at $9000 \mathrm{~m}$ (dashed, $5 \mathrm{~m} \mathrm{~s}^{-1}$ ) computed by the reference model at day 10

formulation for the Prandtl numbers (Cuxart et al. 1995). The parametrization of surface processes is the well-known ISBA scheme (Noilhan and Planton, 1989), allowing for a detailed representation of the effects of various vegetation and soil properties. A warm microphysics parametrization following Kessler (1969) has been introduced. A parametrization of the ice phase has also been developed for three categories of ice crystals, and the code is now under quantitative testing. A deep convection parametrization has been introduced following Kain and Fritsch (1993). Finally, the ECMWF 
radiation code (Morcrette, 1989) has been interfaced with the model.

A chain of programs to initialize MesoNH from actual meteorological analyses has also been developed. Currently, it is possible to initialize simulations with the operational products or the special reanalysis projects from either Meteo-France or the ECMWF. The model should soon include the very important capability of interactive grid-nesting. This will allow us to realize simulataneous simulations of large domains with low resolution, and smaller domains with higher resolution, with two-way interactions between the two domains. Up to eight simultaneous computation domains are being considered in this development. The model also includes a chemical module, allowing for an arbitrary number of reactions with kinetic constants to specify. Recent tests have shown the capability of the model to treat correctly systems of reasonable size. The model allows for detailed budget computation and accumulation of terms during segments of a simulation for any quantity of interest (Hereil, 1996). This tool is believed to offer an interesting potential for future process studies. Quick access to the results is provided by an extensive postprocessing software. This part of the system is rapidly growing and will offer soon an interactive capability. From the practical point of view, the code is written in Fortran90, and the source libraries are managed under the SCCS system, which is available of any UNIX system. All model functions are controlled by name lists, avoiding the need of frequent compilations.

The relevancy of the Lipps and Hemler formulation at very large scale has been addressed in some recent work of the group. As already noted, the definition of the pressure function Eq. (13) includes a significant approximation. It has a beneficial effect for the treatment of stratospheric gravity waves but may be questioned when large simulation domains are used, because the potential temperature may then depart very strongly from the reference state. We have implemented alternative solutions based on the "pseudo-anelastic system" of Durran (1989) and the well-known "modified anelastic system" of Wilhelmson and Ogura (1972). With these formulations, we were indeed able to note an improvement in the absolute value of the pressure diagnosed from the eliptic equation when large domains are used. However, the characteristic growth of error with time seems to be fairly comparable with all three systems, and no conclusion has yet been reached regarding which of the systems is preferable. The implementation of the full compressible system is also considered for the future.

In conclusion, we would like to stress that the current state of the Meso-NH code has nearly reached the initial objective of the project, i.e. to develop a single tool allowing for research in a wide range of topics and atmospheric scales. The validation cases discussed demonstrate that a single code is able to produce state-of-theart results for a range of grid size from $1 \mathrm{~m}$ to $100 \mathrm{~km}$. The verification of the ability of our anelastic system to represent baroclinic waves seems particularly important in this respect. Other tests have demonstrated that correct behaviour of the model over small-scale obstacles, with slopes reaching 70\% . Moreover, several original developments were achieved during this work. The control of the absolute pressure by the boundary conditions, presented in Sect. 2.10, appears to have been achieved for the first time in an anelastic model. The pressure solver, based on a combination of preconditioning by the solution of the flat problem and the traditional Richardson technique, appears to be efficient and accurate. The fluxcorrected advection scheme also has an interesting quality-cost ratio. The combination of various levels of approximation, geometries, advection schemes, initial and boundary conditions offered by Meso-NH also appears rather original. Future developments will further increase the range of possible applications of this unique tool.

Acknowledgements. It would be difficult to give a complete list of people involved in the development or the validation of the Meso$\mathrm{NH}$ model; we would like to express our sincere appreciation to all these colleagues. Without their help, the model would not exist, or would rapidly go out of use. Special thanks to P. Venzac, who produced the simulation of aircraft wake vortex, and to $F$. Lalaurette for his help with the baroclinic wave simulation. The review of this paper by M. Xue was very helpful. The Meso-NH project is supported by Meteo-France and by the Centre National de la Recherche Scientifique (Program PATOM of Institut National des Sciences de l'Univers).

Topical Editor L. Eymard thanks M. Xue for his help in evaluating this paper.

\section{Appendix: Mathematical symbols}

$\vec{\Omega} \quad$ rotation velocity of the earth

$T$ temperature

$P \quad$ absolute pressure

$P_{00} \quad$ reference value of the absolute pressure (100000 $\mathrm{Pa})$

$\rho \quad$ total density of the moist air

$\rho_{d} \quad$ density of the dry fraction of the air

$\rho_{d r e f}$ density of the dry fraction of the air in the reference state

$C_{p d} \quad$ specific heat at constant pressure of dry air

$C_{p v} \quad$ specific heat at constant pressure of water vapour

$C_{l} \quad$ specific heat of liquid water

$C_{i} \quad$ specific heat of ice water

$R_{d} \quad$ gas constant for dry air

$R_{v} \quad$ gas constant for water vapour

$L_{v} \quad$ latent heat of vapourization

$L_{i} \quad$ latent heat of sublimation

$L_{m} \quad$ latent heat of melting

$\Phi$ pressure function

$r_{*} \quad$ mixing ratio of water substance

$s_{*} \quad$ mixing ratio of other scalar

$u, v, w$ Cartesian components of wind

$\Pi \quad$ Exner function

$\Pi_{r e f}$ Exner function of the reference state

() $)_{\text {ref }}$ index signalling the reference state

() $)_{L S}$ index signalling the large-scale state

$\theta$ potential temperature

$\theta_{\text {vref }}$ potential temperature of the reference state

$a$ earth's radius

$r \quad$ distance from the earth's centre 
$\lambda \quad$ longitude

$\phi \quad$ latitude

$\gamma \quad$ angle between current $x$ coordinate and east

$-\beta \quad$ value of $\gamma$ at reference latitude and longitude

$m$ map scale factor

\section{References}

Arakawa, A., and F. Mesinger, Numerical methods used in atmospheric models, GARP Tech. Rep. 17, 1976.

Benoit R., M. Desgagné, P. Pellerin, S. Pellerin, S. Desjardin, and Y. Chartier, The Canadian MC2: a semi-lagrangian, semi-implicit wide-band atmospheric model suited for fine-scale process studies and simulation, Mon. Weather Rev., to appear, 1997

Bernardet, P., The pressure term in the anelastic model: a symmetric elliptic solver for a Arakawa-C grid in generalized coordinates, Mon. Weather Rev., 123, 2474-2490, 1995.

Bougeault, P., B. Bret, P. Lacarrère, and J. Noilhan, An experiment with an advanced surface parameterization in a meso-beta scale model. Part II: the 16 june 1986 simulation, Mon. Weather Rev., 119, 2374-2392, 1991.

Bougeault, P., S. Bélair, S.M. Carrière, J. Cuxart, V. Ducrocq, C. Fischer, P. Héreil, J. P. Lafore, P. Mascart, V. Masson, J.P. Pinty, E. Richard and J. Stein. The Meso-NH atmospheric simulation system: scientific documentation, Météo-France and CNRS, 1996.

Carpenter, K. M., Note on the paper: Radiation conditions for lateral boundaries of limited area numerical models, $Q$. J. R. Meteorol. Soc., 110, 717-719, 1982.

Clark, T. L., A small-scale dynamic model using a terrainfollowing coordinate transformation, J. Comput. Phys., 24, 186-215, 1977.

Cuxart, J., P. Bougeault, and J. L. Redelsperger, Turbulence closure for a non-hydrostatic model, in 11th AMS Symposium on boundary layers and Turbulence, AMS, pp. 409-412, 1995.

Davies, H. C., A lateral boundary formulation for multi-level prediction models, Q. J. R. Meteorol Soc., 102, 405-418, 1976.

Dudhia, J., A nonhydrostatic version of the Penn-State-NCAR mesoscale model: validation tests and simulation of an Atlantic cyclone and cold front, Mon. Weather Rev., 121, 1493-1513, 1993.

Durran, D. R., Improving the anelastic approximation, J. Atmos. Sci., 46, 1453-1461, 1989.

Gal-Chen, T., and R. C. J. Sommerville, On the use of a coordinate transformation for the solution of the navier-stokes equations, J. Comput. Phys., 17, 209-228, 1975.

Golub, G. H., and G. A. Meurant, Résolution des grands systèmes linéaires, Eyrolles, Paris, 1983.

Héreil, P., Dévelopment et tests d'un modèle non hydrostatique pour les ecoulements atmosphériques de méso-Echelle sur relief, These de l'Université Paul Sabatier, Toulouse 1996.

Hoskins, B. J., and N. West, Baroclinic waves and frontogenesis. Part II: uniform potential vorticity jet flows - cold and warm fronts, J. Atmos. Sci., 36, 1663-1680, 1979.

Kain, J. S., and J. M. Fritsch, Convective parametrization for mesoscale models: the Kain-Fritsch Scheme, In: Meteorol. Monogr. Ed. 46, K. Emanuel editor AMS, pp. 165-170, 1993.

Kapitza, H., and D. P. Eppel, The non-hydrostatic mesoscale model GESIMA. Part I: dynamical equations and tests, Cont. Atmos. Phys., 65, 129-146, 1992.

Kessler, E., On the distribution and continuity of water substance in atmospheric circulation, Meteorol. Monogr. 10, 32, 1969.

Klemp, J. B., and R. B. Wilhelmson, The simulation of threedimensional convective storm dynamics, J. Atmos. Sci., 35, 1070-1096, 1978.
Lalaurette, F., C. Fischer, and J.P. Cammas, Location and interaction of upper-air and surface frontogenesis, Mon. Weather Rev., 122, 2004-2021, 1994.

Lipps, F. B., and R. S. Hemler, A scale analysis of deep moist convection and some related numerical calculations, J. Atmos. Sci., 39, 2192-2210, 1982.

Morcrette, J. J., Physical parametrization of the ECMWF Model, ECMWF, Reading.

Nance, L. B., and D. R. Duran, A comparison of three anelastic systems and the pseudo-incompressible system, J. Atmos. Sci., 51, 3549-3565, 1994.

Nickerson, E. C., E. Richard, R. Rosset, and D. R. Smith, The numerical simulation of clouds, rain and airflow over the vosges and black forest mountains: a meso-beta model with parameterised microphysics, Mon. Weather Rev., 114, 398-414, 1986.

Noilhan, J., and S. Planton, A simple parameterization of land surface processes for meteorological models, Mon. Weather Rev., 117, 536-549, 1989.

Orlanski, I., A simple boundary condition for unbounded hyperbolic flows, J. Comput. Phys., 21, 251-269, 1976.

Phillips, N. A., The equations of motions for a shallow rotating atmosphere and the traditional approximation, J. Atmos. Sci., 23, 626, 1966.

Polavarapu, S. M., and W. R. Peltier, The structure and nonlinear evolution of synoptic scale cyclones: life cycle simulations with a cloud-scale model, J. Atmos. Sci., 47, 2645-2672, 1990.

Redelsperger, J. L., and J. P. Lafore, A three-dimensional simulation of a tropical squall-line: convective organization and thermodynamic vertical transport, J. Atmos. Sci., 45, 13341356, 1988.

Saffman, P. G., The approach of a vortex pair to a plane surface in inviscid fluid, J. Fluid Mech, 92, 497-503, 1979.

Scinnoca, J. F., and T. G. Shepherd, Nonlinear wave activity conservation laws and Hamiltonian structure for the twodimensional anelastic equations, J. Atmos. Sci., 49, 5-27, 1992.

Smith, R. B., Linear theory of stratified flow past an isolated mountain, Tellus. 32, 348-364, 1980.

Smolarkiewicz, P. K., The multi-dimensional Crowley advection scheme, Mon. Weather Rev., 110, 1968-1982, 1982.

Smolarkiewicz, P. K., Comment on "A positive definite advection scheme obtained by nonlinear renormalization of the advective fluxes", Mon. Weather Rev., 117, 2626-2632.

Smolarkiewicz, P. K., and W. W. Grabowski, The multidimensional positive definite advection transport algorithm: nonoscilltory option, J. Comput. Phys., 86, 355-375, 1990.

Snyder, C., W. C. Skamarock and R. Rotunno, A comparison of primitive equation and semi-geostrophic simulations of baroclinic waves, J. Atmos. Sci., 48, 2179-2194, 1991.

Staniforth, A., J. Coté , and J. Pudykiewicz, Comments on Smolarkiewicz deformational flow, Mon. Weather Rev., 115, 894-900, 1987.

Viviand, H., Formes conservatives des équations de la dynamique des gaz, Rech. Aerosp, 1, 65-66, 1974.

Wilhelmson, R., and Y. Ogura, The pressure perturbation and the numerical modeling of a cloud, J. Atmos. Sci., 29, 1295-1307, 1972.

Williams, G. P., Numerical integration of the three-dimensional Navier-Stokes equations for incompressible flow, J. Fluid Mech., 37, 727-750, 1969.

Xue, M., K. K. Droegemeier, V. Wong, A. Shapiro, and K. Brewster, ARPS version 4.0 user's guide Center for Analysis and Prediction of Storms, 1995, Univ Oklahoma, 100 E Boyd St. Norman OK 73019.

Xue, M., and A. J. Thorpe, A mesoscale numerical model using the non-hydrostatic pressure-based sigma coordinate equations: model experiments with dry mountain flows, Mon. Weather Rev., 119, 1168-1185, 1991.

Zalesak, S. T., Fully multidimensional flux-corrected transport algorithms for fluids. J. Comput. Phys., 31, 335-362, 1979. 